\title{
Atomic data for the K-vacancy states of Fe XXIV
}

\author{
M. A. Bautista ${ }^{1}$, C. Mendoza ${ }^{2, \star}$, T. R. Kallman ${ }^{2}$, and P. Palmeri ${ }^{2, \star \star}$ \\ 1 Centro de Física, Instituto Venezolano de Investigaciones Científicas (IVIC), PO Box 21827, Caracas 1020A, Venezuela \\ 2 NASA Goddard Space Flight Center, Code 662, Greenbelt, MD 20771, USA
}

Received 26 November 2002 / Accepted 12 March 2003

\begin{abstract}
As part of a project to compute atomic data for the spectral modeling of iron $\mathrm{K}$ lines, we report calculations and comparisons of atomic data for K-vacancy states in Fe XXIV. The data sets include: (i) energy levels, line wavelengths, radiative and Auger rates; (ii) inner-shell electron impact excitation rates and (iii) fine structure inner-shell photoabsorption cross sections. The calculations of energy levels and radiative and Auger rates have involved a detailed study of orbital representations, core relaxation, configuration interaction, relativistic corrections, cancellation effects and semi-empirical corrections. It is shown that a formal treatment of the Breit interaction is essential to render the important magnetic correlations that take part in the decay pathways of this ion. As a result, the accuracy of the present $A$-values is estimated at $10 \%$ while that of the Auger rates at $15 \%$. The calculations of collisional excitation and photoabsorption cross sections take into account the effects of radiation and spectator Auger dampings. In collisional excitation, these effects cause significant attenuation of resonances leading to a good agreement with a simpler method where resonances are excluded. In photoabsorption, resonances converging to the $\mathrm{K}$ thresholds display symmetric profiles of constant width that causes edge smearing.
\end{abstract}

Key words. atomic data - atomic processes - line: formation - X-rays: general

\section{Introduction}

The iron $\mathrm{K}$ lines are among the most interesting features in astronomical X-ray spectra. These lines appear in emission in almost all natural X-ray sources, they are located in a relatively unconfused spectral region and have a well-known plasma diagnostics potential. They were first reported in the rocket observations of the supernova remnant Cas A (Serlemitsos et al. 1973), in X-ray binaries (Sanford et al. 1975; Pravdo et al. 1977) and in clusters of galaxies (Serlemitsos et al. 1977), the latter thus manifesting the presence of extragalactic nuclear processed material. Observations of the galactic blackhole candidate Cyg X-1 showed that the line strength varied according to the spectral state (Barr et al. 1985; Marshall et al. 1993), and Tanaka et al. (1995) found that the Fe K lines from Seyfert galaxies were relativistically broadened and redshifted which suggested their formation within a few gravitational radii of a black hole.

Recent improvements in the spectral capabilities and sensitivity of satellite-borne X-ray telescopes (Chandra, XMMNewton) have promoted the role of Fe K lines in diagnostics, a trend that will continue to grow with the launch of future instruments such as Astro-E2 and Constellation-X. Such plasma

Send offprint requests to: T. R. Kallman, e-mail: timothy.r.kallman@nasa.gov

* Present address: Centro de Física, IVIC, Caracas 1020A.

$\star \star$ Research Associate, Department of Astronomy, University of Maryland, College Park, MD 20742. diagnostics ultimately rely on the knowledge of the microphysics of line formation and hence on the accuracy of the atomic data. In spite of the line identifications by Seely et al. (1986) in solar flare spectra and the laboratory measurements of Beiersdorfer et al. (1989, 1993), Decaux \& Beiersdorfer (1993) and Decaux et al. (1995, 1997), the K-vacancy level structures of Fe ions remain incomplete as can be concluded from the critical compilation of Shirai et al. (2000). With regards to the radiative and Auger rates, the highly ionized members of the isonuclear sequence, namely Fe XVIII-Fe XXV, have received much attention (Jacobs et al. 1989), and the comparisons by Chen (1986) and Kato et al. (1997) have brought about some degree of data assurance. For Fe ions with electron occupancies greater than 9, Jacobs et al. (1980) and Jacobs \& Rozsnyai (1986) have carried out central field calculations on the structure and widths of various inner-shell transitions, but these have not been subject to independent checks and do not meet current requirements of level-to-level data.

The spectral modeling of $\mathrm{K}$ lines also requires accurate knowledge of inner-shell electron impact excitation rates and, in the case of photoionized plasmas, of partial photoionization cross sections leaving the ion in photoexcited K-vacancy states. In this respect, Palmeri et al. (2002) have shown that the K-threshold resonance behavior is dominated by radiation and Auger dampings which induce a smeared edge. Spectator Auger decay, the main contributor of the K-resonance width, has been completely ignored in most previous close-coupling calculations of high-energy continuum processes in $\mathrm{Fe}$ ions 
(Berrington et al. 1997; Donnelly et al. 2000; Berrington \& Ballance 2001; Ballance et al. 2001). The exception is the recent $R$-matrix computation of electron excitation rates of $\mathrm{Li}$ like systems by Whiteford et al. (2002) where it is demonstrated that Auger damping is important for low-temperature effective collision strengths.

The present report is the first in a project to systematically compute atomic data sets for the modeling of the Fe K spectra. The emphasis of this project is on both accuracy and completeness. For this purpose we make use of several state-of-the-art atomic physics codes to deliver for the entire Fe isonuclear sequence: energy levels; wavelengths, radiative and Auger rates, electron impact excitation and photoabsorption cross sections.

In this paper we present calculations of the radiative and Auger decay manifold of the $n=2 \mathrm{~K}$-vacancy states, the innershell electron impact excitation rates of Fe XXIV, and the total photoionization cross sections of Fe XXIII of Fe XXIV, as a test case of the numerical methods and the relevance of various different physical effects. Our goal in doing this is threefold: (i) To understand the competing physical effects which play a significant role in the K vacancy states of iron ions; (ii) To calculate as accurately as possible the atomic data needed for astrophysical modelling of Fe XXIV; (iii) To develop tools which can be applied to the rest of the Fe isonuclear sequence, for which experimental tests and other calculations are less plentiful. Particular attention is given to the process of assessing accuracy and consistency of the data sets produced with respect to experiment and other calculations. We will show that, in comparison with previous work, there is room for improvement. We will also show that by comparison of calculations which incorporate various treatments of the atomic structure and scattering problem it is possible to disentangle and identify the relative importance of the physical processes which affect the accuracy of any calculation.

\section{Breit-Pauli Hamiltonian}

We have found the Li-like Fe system to be an unusually versatile workbench for the magnetic interactions, a fact that perhaps has not been fully appreciated in previous work. Thus prior to the description of the numerical details of the codes, we include a concise summary of the relativistic Breit-Pauli Hamiltonian which is used throughout our computational portfolio and will be central in the discussion of results.

The Breit-Pauli Hamiltonian for an $N$-electron system is given by

$H_{\mathrm{bp}}=H_{\mathrm{nr}}+H_{1 \mathrm{~b}}+H_{2 \mathrm{~b}}$

where $H_{\mathrm{nr}}$ is the usual non-relativistic Hamiltonian. The onebody relativistic operators

$H_{1 \mathrm{~b}}=\sum_{n=1}^{N} f_{n}($ mass $)+f_{n}(\mathrm{~d})+f_{n}(\mathrm{so})$

represent the spin-orbit interaction, $f_{n}(\mathrm{so})$, and the nonfine structure mass-variation, $f_{n}$ (mass), and one-body Darwin, $f_{n}(\mathrm{~d})$, corrections. The two-body corrections

$H_{2 \mathrm{~b}}=\sum_{n>m} g_{n m}(\mathrm{so})+g_{n m}(\mathrm{ss})+g_{n m}(\mathrm{css})+g_{n m}(\mathrm{~d})+g_{n m}(\mathrm{oo}),(3)$ usually referred to as the Breit interaction, include, on the one hand, the fine structure terms $g_{n m}$ (so) (spin-other-orbit and mutual spin-orbit) and $g_{n m}($ ss) (spin-spin); and on the other, the non-fine structure terms: $g_{n m}(\mathrm{css})$ (spin-spin contact), $g_{n m}(\mathrm{~d})$ (Darwin) and $g_{n m}($ oo) (orbit-orbit).

The radiative rates ( $A$-values) for electric dipole and quadrupole transitions are respectively given in units of $\mathrm{s}^{-1}$ by the expressions

$$
\begin{aligned}
& A_{\mathrm{E} 1}(k, i)=2.6774 \times 10^{9}\left(E_{k}-E_{i}\right)^{3} \frac{1}{g_{k}} S_{\mathrm{E} 1}(k, i) \\
& A_{\mathrm{E} 2}(k, i)=2.6733 \times 10^{3}\left(E_{k}-E_{i}\right)^{5} \frac{1}{g_{k}} S_{\mathrm{E} 2}(k, i)
\end{aligned}
$$

where $S(k, i)$ is the line strength, $g_{k}$ is the statistical weight of the upper level, and energies are in Rydberg units and lengths in Bohr radii.

Similarly for magnetic dipole and quadrupole transitions, the $A$-values are respectively given by

$$
\begin{aligned}
& A_{\mathrm{M} 1}(k, i)=3.5644 \times 10^{4}\left(E_{k}-E_{i}\right)^{3} \frac{1}{g_{k}} S_{\mathrm{M} 1}(k, i) \\
& A_{\mathrm{M} 2}(k, i)=2.3727 \times 10^{-2}\left(E_{k}-E_{i}\right)^{5} \frac{1}{g_{k}} S_{\mathrm{M} 2}(k, i) .
\end{aligned}
$$

Due to the strong magnetic interactions in this ion, the magnetic dipole line strength is assumed to take the form

$S_{\mathrm{M} 1}(k, i)=|\langle|k| \boldsymbol{P} \mid i\rangle|^{2}$

where

$\boldsymbol{P}=\boldsymbol{P}^{0}+\boldsymbol{P}^{1}=\sum_{n=1}^{N}\{\boldsymbol{l}(n)+\sigma(n)\}+\boldsymbol{P}^{\mathrm{rc}}$.

$\boldsymbol{P}^{0}$ is the usual low-order M1 operator while $\boldsymbol{P}^{\text {rc }}$ includes the relativistic corrections established by Drake (1971).

Although the main astrophysical interest is for $\mathrm{E} 1 \mathrm{~K} \alpha$ decays, it is shown here that some of the forbidden transitions display $A$-values comparable with the E1 type and thus must be taken into account for accuracy. Furthermore, in the case of the $1 \mathrm{~s} 2 \mathrm{~s} 2 \mathrm{p}{ }^{4} \mathrm{P}_{5 / 2}^{0}$ state, radiative decay can only occur through forbidden transitions.

\section{Numerical methods}

In the present work we employ three different computational packages to study the properties of the $n=2$ vacancy states of Li-like Fe XXIV.

\subsection{AUTOSTRUCTURE}

AUTOSTRUCTURE, an extension by Badnell (1986, 1997) of the atomic structure program SUPERSTRUCTURE (Eissner et al. 1974), computes fine-structure level energies, radiative and Auger rates in a Breit-Pauli relativistic framework. Single electron orbitals, $P_{n l}(r)$, are constructed by diagonalizing the non-relativistic Hamiltonian, $H_{\mathrm{nr}}$, within a statistical Thomas-Fermi-Dirac model potential $V\left(\lambda_{n l}\right)$ (Eissner \& Nussbaumer 1969). The $\lambda_{n l}$ scaling parameters are 
optimized variationally by minimizing a weighted sum of the $L S$ term energies. $L S$ terms are represented by configurationinteraction $(\mathrm{CI})$ wavefunctions of the type

$\Psi(L S)=\sum_{i} c_{i} \phi_{i}$

Continuum wavefunctions are constructed within the distortedwave approximation. Relativistic fine-structure levels and rates are obtained by diagonalizing the Breit-Pauli Hamiltonian in intermediate coupling. The one- and two-body operators - fine structure and non-fine structure (see Sect. 2) - have been fully implemented to order $\alpha^{2} Z^{4}$ where $\alpha$ is the fine-structure constant and $Z$ the atomic number. The relativistic corrections to the M1 operator (see Eq. (9)) have been incorporated in SUPERSTRUCTURE by Eissner \& Zeippen (1981).

Fine tuning (semi-empirical corrections) - which is resourceful for treating states that decay through weak relativistic couplings (e.g. intercombination transitions) - takes the form of term energy corrections (TEC). By considering the relativistic wavefuntion, $\psi_{i}^{\mathrm{r}}$, in an perturbation expansion of the nonrelativistic functions $\psi_{i}^{\mathrm{nr}}$,

$\psi_{i}^{\mathrm{r}}=\psi_{i}^{\mathrm{nr}}+\sum_{j \neq i} \psi_{j}^{\mathrm{nr}} \times \frac{\left\langle\psi_{j}^{\mathrm{nr}}\left|H_{1 \mathrm{~b}}+H_{2 \mathrm{~b}}\right| \psi_{i}^{\mathrm{nr}}\right\rangle}{E_{i}^{\mathrm{nr}}-E_{j}^{\mathrm{nr}}}$,

a modified $H_{n r}$ is constructed with improved estimates of the differences $E_{i}^{\mathrm{nr}}-E_{j}^{\mathrm{nr}}$ so as to adjust the centers of gravity of the spectroscopic terms to the experimental values. This procedure therefore relies on the availability of measured data.

\subsection{HFR}

In the Hartree-Fock plus relativistic corrections code (HFR) by Cowan (1981), a set of orbitals are obtained for each electronic configuration by solving the Hartree-Fock equations for the spherically averaged atom. The equations are the result of the application of the variational principle to the configuration average energy. Relativistic corrections are also included in this set of equations, i.e. the Blume-Watson spin-orbit, massvariation and one-body Darwin terms. The Blume-Watson spin-orbit term comprises the part of the Breit interaction that can be reduced to a one-body operator.

The multiconfiguration Hamiltonian matrix is constructed and diagonalized in the $L S J \pi$ representation within the framework of the Slater-Condon theory. Each matrix element is a sum of products of Racah angular coefficients and radial integrals (Slater and spin-orbit integrals), i.e.

$\langle a|H| b\rangle=\sum_{i} c_{i}^{a, b} I_{i}^{a, b}$

The radial parameters, $I_{i}^{a, b}$, can be adjusted to fit the available experimental energy levels in a least-squares approach. The eigenvalues and the eigenstates obtained in this way ( $\mathrm{ab}$ initio or semi-empirically) are used to compute the wavelength and oscillator strength for each possible transition.
Autoionization rates can be calculated using the perturbation approach

$$
\begin{aligned}
A^{a} & =\frac{2 \pi}{\hbar} V_{\varepsilon}^{2} \\
& =\frac{2 \pi}{\hbar}|<\alpha L S J \pi| H\left|\alpha^{\prime} L^{\prime} S^{\prime} J^{\prime} \varepsilon l L S J \pi>\right|^{2}
\end{aligned}
$$

where $\alpha$ summarizes the coupling scheme and the remaining set of quantum numbers necessary to define the initial state, and $\alpha^{\prime}$ plays a similar role for the threshold state to which the continuum electron, $\varepsilon l$, is coupled. The kinetic energy of the free electron, $\varepsilon$, is determined as the difference between the average energy of the autoionizing and the threshold configurations. The radial wave functions of the initial and final states are optimized separately. Both states are calculated in intermediate coupling but $\mathrm{CI}$ is accounted for only in the autoionizing states, i.e. no interaction between threshold electronic configurations is introduced. The continuum orbitals, $P_{\varepsilon l}(r)$, are solutions of the Hartree-Plus-Statistical-Exchange equations for fixed positive values of the Lagrangian multipliers, $\varepsilon$ (Cowan 1981).

\subsection{BPRM}

The Breit-Pauli $R$-matrix package (BPRM) is widely used in electron-ion scattering and in radiative bound-bound and bound-free calculations. It is based of the close-coupling approximation of Burke \& Seaton (1971) whereby the wavefunctions for states of an $N$-electron target and a colliding electron with total angular momentum and parity $J \pi$ are expanded in terms of the target eigenfunctions

$$
\Psi^{J \pi}=\mathcal{A} \sum_{i} \chi_{i} \frac{F_{i}(r)}{r}+\sum_{j} c_{j} \Phi_{j} .
$$

The functions $\chi_{i}$ are vector coupled products of the target eigenfunctions and the angular components of the incidentelectron functions, $F_{i}(r)$ are the radial part of the latter and $\mathcal{A}$ is an antisymmetrization operator. The functions $\Phi_{j}$ are boundtype functions of the total system constructed with target orbitals; they are introduced to compensate for orthogonality conditions imposed on the $F_{i}(r)$ and to improve short-range correlations. The Kohn variational gives rise to a set of coupled integro-differential equations that are solved by $R$-matrix techniques (Burke et al. 1971; Berrington et al. 1974, 1978, 1987) within a box of radius, say, $r \leq a$. In the asymptotic region $(r>a)$ exchange between the outer electron and the target ion can be neglected, and the wavefunctions can be then approximated by Coulomb solutions. Resonance parameters are obtained with the STGQB module developed by Quigley \& Berrington (1996) and Quigley et al. (1998) whereby the resonance positions and widths are obtained from fits of the eigenphase sum. Normalized partial widths are defined from projections onto the open channels.

Breit-Pauli relativistic corrections have been introduced in the $R$-matrix suite by Scott \& Burke (1980), Scott \& Taylor (1982), but the two-body terms (see Eq. (3)) have not as yet been implemented. Inter-channel coupling is equivalent to $\mathrm{CI}$ 
Table 1. Ion model key. AST1-AST3: present work (AUTOSTRUCTURE). HFR1-HFR3: Present work (HFR). HFR4: HFR calculation by Jacobs et al. (1989). BPR1: Present work (BPRM). COR: Cornille data set from Kato et al. (1997). SAF: Safronova data set from Kato et al. (1997) and Safronova \& Shlyaptseva (1996). MCDF: Multiconfiguration Dirac-Fock calculation by Chen (1986).

\begin{tabular}{llllllllllll}
\hline \hline Feature & AST1 & AST2 & AST3 & HFR1 & HFR2 & HFR3 & HFR4 & BPR1 & COR & SAF & MCDF \\
\hline Orthogonal orbital basis & Yes & Yes & Yes & Yes & No & No & No & Yes & Yes & Yes & Yes \\
CI from $n>2$ complexes & No & No & Yes & No & No & Yes & No & No & No & Yes & Yes \\
Breit interaction & No & Yes & Yes & Yes & Yes & Yes & Yes & No & No & Yes & Yes \\
QED effects & No & No & No & No & No & No & No & No & No & Yes & Yes \\
Semi-empirical corrections & No & No & Yes & No & No & Yes & No & No & No & No & No \\
\hline
\end{tabular}

in the atomic structure context, and thus the BPRM method presents a formal and unified approach to study the decay properties of both bound states and resonances.

\section{Radiation and Auger dampings}

When an electron or a photon are sufficiently energetic to excite a ground-state ion to a K-vacancy resonance, the latter can either fluoresce or autoionize (Auger decay). Illustrating these processes with the resonances converging to the $n=2$ $\mathrm{K}$ thresholds in the dielectronic recombination of Fe XXIV and the photoexcitation of Fe XXIII, that is

$$
\left\{\begin{array}{l}
\mathrm{Fe}^{23+}\left(1 \mathrm{~s}^{2} 2 \mathrm{~s}\right)+\mathrm{e}^{-} \\
\mathrm{Fe}^{22+}\left(1 \mathrm{~s}^{2} 2 \mathrm{~s}^{2}\right)+h v
\end{array}\right\} \rightarrow\left\{\begin{array}{l}
\mathrm{Fe}^{22+}\left(1 \mathrm{~s} 2 \mathrm{~s}^{2} n l\right) \\
\mathrm{Fe}^{22+}(1 \mathrm{~s} 2 \mathrm{~s} 2 \mathrm{p} n l) \\
\mathrm{Fe}^{22+}\left(1 \mathrm{~s} 2 \mathrm{p}^{2} n l\right)
\end{array}\right\}
$$

the decay manifold can be outlined as follows:

$$
\begin{aligned}
\left\{\begin{array}{l}
\mathrm{Fe}^{22+}\left(1 \mathrm{~s} 2 \mathrm{~s}^{2} n l\right) \\
\mathrm{Fe}^{22+}(1 \mathrm{~s} 2 \mathrm{~s} 2 \mathrm{p} n l) \\
\mathrm{Fe}^{22+}\left(1 \mathrm{~s} 2 \mathrm{p}^{2} n l\right)
\end{array}\right\} & \rightarrow\left\{\begin{array}{l}
\mathrm{Fe}^{23+}\left(1 \mathrm{~s}^{2} 2 \mathrm{~s}\right)+\mathrm{e}^{-} \\
\mathrm{Fe}^{23+}\left(1 \mathrm{~s}^{2} 2 \mathrm{p}\right)+\mathrm{e}^{-}
\end{array}\right\} \\
& \rightarrow\left\{\mathrm{Fe}^{23+}\left(1 \mathrm{~s}^{2} n l\right)+\mathrm{e}^{-}\right\} \\
& \rightarrow\left\{\begin{array}{l}
\mathrm{Fe}^{22+}\left(1 \mathrm{~s}^{2} 2 \mathrm{~s}^{2}\right)+h v \\
\mathrm{Fe}^{22+}\left(1 \mathrm{~s}^{2} 2 \mathrm{~s} 2 \mathrm{p}\right)+h v \\
\mathrm{Fe}^{22+}\left(1 \mathrm{~s}^{2} 2 \mathrm{p}^{2}\right)+h v
\end{array}\right\} \\
& \rightarrow\left\{\begin{array}{l}
\mathrm{Fe}^{22+}\left(1 \mathrm{~s}^{2} 2 \mathrm{~s} n l\right)+h v \\
\mathrm{Fe}^{22+}\left(1 \mathrm{~s}^{2} 2 \mathrm{p} n l\right)+h v
\end{array}\right\}
\end{aligned}
$$

The participator KLn Auger channels (Eq. (16)) can be adequately represented in the BPRM method by including in the close-coupling expansion (14) configuration-states within the $n=2$ complex of the three-electron target. On the other hand, in the KLL Auger process in Eq. (17), also referred to as spectator Auger decay, the $n l$ Rydberg electron remains a spectator. Its formal handling in the close-coupling approach is thus severely limited to low- $n$ resonances as it implies the inclusion of target states with $n l$ orbitals. Moreover, it has been recently shown by Palmeri et al. (2002) that KLL is the dominant Auger decay mode in the Fe sequence by no less than $75 \%$, and leads to photoabsorption cross sections populated with damped resonances of constant widths as $n \rightarrow \infty$ which causes the smearing of the edge.

Transitions in Eqs. (18) and (19) lead to radiation damping. The former, to be referred to as the $\mathrm{K} n$ transition array, are driven by the $n \mathrm{p} \rightarrow 1 \mathrm{~s}$ optical electron jump. The latter is the $\mathrm{K} \alpha$ transition array $(2 \mathrm{p} \rightarrow 1 \mathrm{~s})$ where again the $n l$ Rydberg electron remains a spectator; its dominant width is therefore practically independent of $n$ (Palmeri et al. 2002).

The present treatment of Auger and radiative dampings within the BPRM framework uses the optical potential described by Gorczyca \& Badnell (1996) and Gorczyca \& Badnell (2000), where the resonance threshold energy acquires an imaginary component. For example, the core energy of the closed channel 1s2s $2 \mathrm{p} n l$ is now expressed as

$E_{1 s^{-1}} \rightarrow E_{1 s^{-1}}-i\left(\Gamma_{1 s^{-1}}^{a}+\Gamma_{1 s^{-1}}^{r}\right) / 2$,

where $\Gamma_{1 s^{-1}}^{a}$ and $\Gamma_{1 s^{-1}}^{r}$ are respectively the Auger and radiative widths of the $1 \mathrm{~s} 2 \mathrm{~s} 2 \mathrm{p}$ core. In the case of radiation damping, the optical potential modifies the $R$-matrix to the complex form

$R_{j j^{\prime}}(E)=R_{j j^{\prime}}^{0}(E)+2 \sum_{n n^{\prime}} d_{j n}^{0} d_{j^{\prime} n^{\prime}}^{0}\left(\gamma^{-1}\right)_{n n^{\prime}}$,

where $R_{j j^{\prime}}^{0}$ are the $R$-matrix elements without damping, $d_{j n}^{0}$ are $(N+1)$-electron dipole matrix elements and $\gamma^{-1}$ is a small inverted complex matrix defined in Eq. (100) of Robicheaux et al. (1995).

The calculations of collisional excitation and photoionization with the BPRM method are carried out with the standard $R$-matrix computer package of Berrington et al. (1995) for the inner region and on the asymptotic codes STGFDAMP (Gorczyca \& Badnell 1996) and STGBF0FAMP (Badnell, unpublished) to determine cross sections including radiation and Auger dampings.

\section{Ion models}

Since the present study of the Fe Li-like system has been approached as a test case, the atomic data are computed with several ion models and extensively compared with other data sets. This methodology is destined to bring out the dominant physical effects and the flaws and virtues of the different numerical packages. Additionally, it provides statistics for determining accuracy ratings, something which has not been fully established in the past. The main features of each approximation are summarized in the key in Table 1.

Three calculations with AUTOSTRUCTURE are listed: AST1, the ion is modeled with states from configurations within the $n=2$ complex and excludes the Breit interaction, i.e. the relativistic two-body operators in Eq. (3); AST2, the same as AST1 but takes into account the Breit interaction; and AST3, which includes the latter, single and double excitations to the $n=3$ complex and TEC. AST3 allows the evaluation of CI effects 
from higher complexes and the fine-tuning of the data for accuracy. Orthogonal orbital bases are generated for each of these three approximations by minimizing the sum of the energies of all the $L S$ terms comprising the respective ion representations. A dilemma quickly arises in AUTOSTRUCTURE calculations regarding the ion model in the context of Auger processes, whether to use Li-type orbitals (parent ion) or those of the Helike remnant. By comparing with results from the more formal BPRM method, it becomes clear that the latter type is the superior choice. On the other hand, the situation is less certain for the $\mathrm{K} \alpha$ radiative data due to the absence of noticeable differences. In this case, and due to better agreement with previous work, the $A$-values have been calculated with parent orbitals.

Three computations with HFR are discussed: HFR1 is equivalent to AST2 since the ion is modeled with states within the $n=2$ complex with an orthogonal orbital basis. The $1 \mathrm{~s}$ and $2 \mathrm{~s}$ orbitals are obtained by minimizing the energy of the $1 \mathrm{~s}^{2} 2 \mathrm{~s}$ term whereas the $2 p$ is optimized with $1 s^{2} 2 p$. HFR2 employs the ion model of HFR1 but with non-orthogonal orbital bases generated for each configuration by minimizing their average energy. Comparisons of HFR1 and HFR2 will thus give estimates of core relaxation effects (CRE) which have been long known (Howat 1978; Howat et al. 1978; Breuckmann 1979) but generally neglected in the more recent work on the $\mathrm{Fe}$ isonuclear sequence. In HFR3 non-orthogonal bases are used, full $n=3 \mathrm{CI}$ is taken into account and the radial integrals are fitted to reproduce experimental energies (this approximation should then be comparable to AST3).

BPR1 is a computation with BPRM wherein the He-like target is represented with the 19 levels from the $1 \mathrm{~s}^{2}, 1 \mathrm{~s} 2 \mathrm{~s}$, and $1 s 2 p$ configurations. Since BPRM does not take into account the Breit interaction, BPR1 should be comparable with AST1.

We also compare with four external data sets (see Table 1). HFR 4 contains wavelengths, radiative rates and satellite line intensity factors computed with HFR by Jacobs et al. (1989); CI is only taken into account within the $n=2$ complex, and therefore this data set would be comparable to our HFR2. COR, corresponds to the data set referred to as "Cornille" in Kato et al. (1997) computed with the program AUTOLSJ (Dubau \& Loulergue 1981), an earlier but similar implementation of AUTOSTRUCTURE. SAF contains the data set "Safronova" in Kato et al. (1997) and energy levels reported in Safronova \& Shlyaptseva (1996) that have been obtained with a $1 / Z$ perturbation method. This method uses a hydrogenic orbital basis, the correlation energy includes contributions from both discrete and continuum states, and the two-body operators of the Breit interaction and QED effects are obtained in a hydrogenic approximation through screening constants. MCDF (Chen 1986) contains data computed in a multiconfiguration Dirac-Fock method that accounts for the Breit interaction and QED in the transition energy, but excludes the exchange interaction between the bound and continuum electrons.

In our comparisons two external computations are excluded. Lemen et al. (1984) have computed Auger rates with HFR in a single configuration approximation (i.e. no CI even within $n=2$ ), the Breit interaction is not taken into account and the Coulomb integrals are empirically scaled by $15 \%$ to allow for neglected effects. The large discrepancies found with our HFR calculations can be perhaps attributed their questionable atomic model. Nahar et al. (2001) have computed with BPRM radiative and Auger widths for the $1 \mathrm{~s} 2 \mathrm{~s} 2 \mathrm{p}$ states. There is good general accord with our BPR1 results, and since they only report a reduced data set, it will not be further discussed.

\section{Energies and wavelengths}

In Table 2 we compare present level energies with experiment and SAF. It may be seen that the energies obtained for the K-vacancy levels with approximation AST1 are on average $10 \pm 2 \mathrm{eV}$ higher than experiment. By including the Breit interaction (AST2), and mainly due to the contribution from the non-fine structure two-body terms, this discrepancy is slightly reduced to $8 \pm 1 \mathrm{eV}$. Further consideration of CI, i.e. from configurations of the $n=3$ complex, does not bring about noticeable improvements. Results obtained with BPR1 bear a similar degree of discord with measured values. This systematic difference is partly due to neglected interactions (e.g. QED), but also to the fact that orthogonal orbital bases are used to represent the ground and lowly excited bound states, in the one hand, and the highly excited K-vacancy resonances on the other thus discarding CRE. This assertion is supported by a comparison of average differences of HFR1 (excludes CRE) and HFR2 (includes CRE) with experiment: $5 \pm 1 \mathrm{eV}$ and $2 \pm 1 \mathrm{eV}$ respectively. Fine tuning, invoked in approximations AST3 and HFR3, results in theoretical levels within $1 \mathrm{eV}$ of experiment, comparable to the accuracy of $1.5 \mathrm{eV}$ displayed by SAF. For the unobserved $1 \mathrm{~s} 2 \mathrm{~s} 2 \mathrm{p}^{4} \mathrm{P}_{5 / 2}^{0}$ level, an energy of $6.6285(3) \mathrm{keV}$ is predicted which is in good accord with value of $6.6283 \mathrm{keV}$ quoted by SAF.

In Table 3 we compare line wavelengths derived from the AST3 and HFR3 approximations with experiment and other theoretical results. The measurements have been made by Beiersdorfer et al. (1993) with a high-resolution Bragg crystal spectrometer on the Princeton Large Torus Tokamak. Our previous criticism regarding the incompleteness of the experimental data sets can be appreciated in this comparison. With respect to experiment, differences with HFR3 and SAF are not larger than $0.4 \mathrm{~m} \AA$ while those with AST3 and MCDF are within $0.6 \mathrm{~m} \AA$ and $0.8 \mathrm{~m} \AA$ respectively. This level of accord is somewhat outside of the average experimental precision of $0.23 \mathrm{~mA}$. The values listed by COR and HFR4 are systematically shorter than experiment by $\sim 3 \mathrm{~m} \AA$ and $\sim 2 \mathrm{~m} \AA$, respectively. In general, differences between the AST3, HFR3, SAF and MCDF data sets show scatters with standard deviations not larger than $0.3 \mathrm{~m} \AA$ which can perhaps be taken as a lower bound of the theoretical accuracy.

\section{Radiative rates}

A Li-like $\mathrm{K}$-vacancy state decays radiatively by emitting a $\mathrm{K} \alpha$ photon

$1 \mathrm{~s} 2 \mathrm{~s}^{n_{k}} 2 \mathrm{p}^{m_{k}\left(2 S_{k}+1\right)} L_{J_{k}} \rightarrow 1 \mathrm{~s}^{2} 2 l_{i}^{2} L_{J_{i}}^{\prime}+\lambda_{\mathrm{K} \alpha}$

where the strong transitions are the dipole spin-allowed $\left(2 S_{k}+\right.$ $1=2)$, but intercombination transitions $\left(2 S_{k}+1=4\right)$ can also take place via subtle relativistic couplings. Furthermore, 
Table 2. Comparison of level energies (keV) for the $n=2$ complex of Fe XXIV (see approximation key in Table 1). Experimental values from Shirai et al. (2000).

\begin{tabular}{|c|c|c|c|c|c|c|c|c|c|c|}
\hline$i$ & State & Expt & AST1 & AST2 & AST3 & HFR1 & HFR2 & HFR3 & BPR1 & SAF \\
\hline 1 & $1 s^{2} 2 s^{2} S_{1 / 2}$ & 0.0 & 0.0 & 0.0 & 0.0 & 0.0 & 0.0 & 0.0 & 0.0 & 0.0 \\
\hline 2 & $1 \mathrm{~s}^{2} 2 \mathrm{p}^{2} \mathrm{P}_{1 / 2}^{0}$ & 0.04860 & 0.04801 & 0.04928 & 0.04778 & 0.04843 & 0.04850 & 0.04860 & & 0.04854 \\
\hline 3 & $1 s^{2} 2 p^{2} P_{3 / 2}^{0}$ & 0.06457 & 0.06696 & 0.06689 & 0.06498 & 0.06446 & 0.06454 & 0.06457 & & 0.06453 \\
\hline 4 & $1 \mathrm{~s} 2 \mathrm{~s}^{2}{ }^{2} \mathrm{~S}_{1 / 2}$ & 6.6004 & 6.6099 & 6.6070 & 6.6003 & 6.6051 & 6.6018 & 6.6004 & 6.6072 & 6.6011 \\
\hline 5 & $1 \mathrm{~s}\left({ }^{2} \mathrm{~S}\right) 2 \mathrm{~s} 2 \mathrm{p}\left({ }^{3} \mathrm{P}^{0}\right){ }^{4} \mathrm{P}_{1 / 2}^{0}$ & 6.6137 & 6.6202 & 6.6189 & 6.6131 & 6.6175 & 6.6129 & 6.6131 & 6.6177 & 6.6135 \\
\hline 6 & $1 \mathrm{~s}\left({ }^{2} \mathrm{~S}\right) 2 \mathrm{~s} 2 \mathrm{p}\left({ }^{3} \mathrm{P}^{0}\right){ }^{4} \mathrm{P}_{3 / 2}^{0}$ & 6.6167 & 6.6253 & 6.6227 & 6.6169 & 6.6221 & 6.6178 & 6.6173 & 6.6230 & 6.6171 \\
\hline 7 & $1 \mathrm{~s}\left({ }^{2} \mathrm{~S}\right) 2 \mathrm{~s} 2 \mathrm{p}\left({ }^{3} \mathrm{P}^{0}\right){ }^{4} \mathrm{P}_{5 / 2}^{0}$ & & 6.6376 & 6.6342 & 6.6285 & 6.6330 & 6.6295 & 6.6265 & & 6.6283 \\
\hline 8 & $1 \mathrm{~s}\left({ }^{2} \mathrm{~S}\right) 2 \mathrm{~s} 2 \mathrm{p}\left({ }^{3} \mathrm{P}^{0}\right){ }^{2} \mathrm{P}_{1 / 2}^{0}$ & 6.6535 & 6.6624 & 6.6598 & 6.6525 & 6.6567 & 6.6538 & 6.6537 & 6.6605 & 6.6534 \\
\hline 9 & $1 \mathrm{~s}\left({ }^{2} \mathrm{~S}\right) 2 \mathrm{~s} 2 \mathrm{p}\left({ }^{3} \mathrm{P}^{0}\right){ }^{2} \mathrm{P}_{3 / 2}^{0}$ & 6.6619 & 6.6732 & 6.6697 & 6.6623 & 6.6665 & 6.6641 & 6.6618 & 6.6708 & 6.6624 \\
\hline 10 & $1 \mathrm{~s}\left({ }^{2} \mathrm{~S}\right) 2 \mathrm{p}^{2}\left({ }^{3} \mathrm{P}\right){ }^{4} \mathrm{P}_{1 / 2}$ & 6.6710 & 6.6781 & 6.6770 & 6.6706 & 6.6753 & 6.6709 & 6.6708 & 6.6764 & 6.6717 \\
\hline 11 & $1 \mathrm{~s}\left({ }^{2} \mathrm{~S}\right) 2 \mathrm{~s} 2 \mathrm{p}\left({ }^{1} \mathrm{P}^{0}\right){ }^{2} \mathrm{P}_{1 / 2}^{0}$ & 6.6764 & 6.6866 & 6.6841 & 6.6764 & 6.6814 & 6.6784 & 6.6766 & 6.6831 & 6.6765 \\
\hline 12 & $1 \mathrm{~s}\left({ }^{2} \mathrm{~S}\right) 2 \mathrm{~s} 2 \mathrm{p}\left({ }^{1} \mathrm{P}^{0}\right){ }^{2} \mathrm{P}_{3 / 2}^{0}$ & 6.6792 & 6.6896 & 6.6867 & 6.6791 & 6.6839 & 6.6812 & 6.6790 & 6.6869 & 6.6795 \\
\hline 13 & $1 \mathrm{~s}\left({ }^{2} \mathrm{~S}\right) 2 \mathrm{p}^{2}\left({ }^{3} \mathrm{P}\right){ }^{4} \mathrm{P}_{3 / 2}$ & 6.6793 & 6.6868 & 6.6855 & 6.6792 & 6.6829 & 6.6790 & 6.6786 & 6.6853 & 6.6798 \\
\hline 14 & $1 \mathrm{~s}\left({ }^{2} \mathrm{~S}\right) 2 \mathrm{p}^{2}\left({ }^{3} \mathrm{P}\right){ }^{4} \mathrm{P}_{5 / 2}$ & 6.6850 & 6.6946 & 6.6917 & 6.6850 & 6.6900 & 6.6865 & 6.6857 & 6.6932 & 6.6856 \\
\hline 15 & $1 \mathrm{~s}\left({ }^{2} \mathrm{~S}\right) 2 \mathrm{p}^{2}\left({ }^{1} \mathrm{D}\right){ }^{2} \mathrm{D}_{3 / 2}$ & 6.7027 & 6.7137 & 6.7118 & 6.7027 & 6.7082 & 6.7050 & 6.7029 & 6.7112 & 6.7042 \\
\hline 16 & $1 \mathrm{~s}\left({ }^{2} \mathrm{~S}\right) 2 \mathrm{p}^{2}\left({ }^{3} \mathrm{P}\right){ }^{2} \mathrm{P}_{1 / 2}$ & 6.7046 & 6.7159 & 6.7128 & 6.7041 & 6.7099 & 6.7068 & 6.7048 & 6.7141 & 6.7052 \\
\hline 17 & $1 \mathrm{~s}\left({ }^{2} \mathrm{~S}\right) 2 \mathrm{p}^{2}\left({ }^{1} \mathrm{D}\right){ }^{2} \mathrm{D}_{5 / 2}$ & 6.7090 & 6.7211 & 6.7176 & 6.7089 & 6.7147 & 6.7120 & 6.7096 & 6.7189 & 6.7097 \\
\hline 18 & $1 \mathrm{~s}\left({ }^{2} \mathrm{~S}\right) 2 \mathrm{p}^{2}\left({ }^{3} \mathrm{P}\right){ }^{2} \mathrm{P}_{3 / 2}$ & 6.7224 & 6.7349 & 6.7315 & 6.7225 & 6.7268 & 6.7247 & 6.7219 & 6.7329 & 6.7230 \\
\hline 19 & $1 \mathrm{~s}\left({ }^{2} \mathrm{~S}\right) 2 \mathrm{p}^{2}\left({ }^{1} \mathrm{~S}\right){ }^{2} \mathrm{~S}_{1 / 2}$ & 6.7415 & 6.7541 & 6.7514 & 6.7414 & 6.7468 & 6.7448 & 6.7412 & 6.7519 & 6.7428 \\
\hline
\end{tabular}

Table 3. Comparison of wavelengths ( $\AA$ ) for K transitions in Fe XXIV (see approximation key in Table 1). Transition labels from Gabriel (1972) and tokamak measurements (uncertainties in brackets) by Beiersdorfer et al. (1993).

\begin{tabular}{crllllllll}
\hline \hline Label & $k$ & $i$ & Expt & AST3 & HFR3 & HFR4 & COR & SAF & MCDF \\
\hline $\mathrm{p}$ & 4 & 2 & $1.89219(25)$ & 1.8922 & 1.8924 & & 1.8894 & 1.8924 & 1.8927 \\
$\mathrm{o}$ & 4 & 3 & $1.89680(20)$ & 1.8971 & 1.8970 & & 1.8946 & 1.8969 & 1.8973 \\
$\mathrm{v}$ & 5 & 1 & & 1.8748 & 1.8748 & 1.8729 & & 1.8748 & 1.8752 \\
$\mathrm{u}$ & 6 & 1 & $1.87347(35)$ & 1.8737 & 1.8736 & 1.8715 & 1.8712 & 1.8738 & 1.8742 \\
& 7 & 1 & & 1.8706 & & & & & \\
& 7 & 3 & & 1.8890 & & & & & \\
$\mathrm{r}$ & 8 & 1 & $1.86325(20)$ & 1.8639 & 1.8634 & 1.8618 & 1.8611 & 1.8635 & 1.8640 \\
$\mathrm{q}$ & 9 & 1 & $1.86104(15)$ & 1.8610 & 1.8611 & 1.8589 & & 1.8610 & 1.8613 \\
$\mathrm{i}$ & 10 & 2 & & 1.8720 & 1.8722 & 1.8706 & & 1.8722 & 1.8725 \\
$\mathrm{~h}$ & 10 & 3 & & 1.8768 & 1.8768 & 1.8752 & & 1.8766 & 1.8771 \\
$\mathrm{t}$ & 11 & 1 & $1.85693(20)$ & 1.8568 & 1.8570 & 1.8552 & 1.8543 & 1.8571 & 1.8571 \\
$\mathrm{~s}$ & 12 & 1 & & 1.8563 & 1.8563 & 1.8544 & 1.8535 & 1.8563 & 1.8564 \\
$\mathrm{~g}$ & 13 & 2 & & 1.8697 & 1.8701 & 1.8680 & & 1.8699 & 1.8702 \\
$\mathrm{f}$ & 13 & 3 & & 1.8745 & 1.8746 & 1.8725 & 1.8724 & 1.8743 & 1.8747 \\
$\mathrm{e}$ & 14 & 3 & $1.87246(35)$ & 1.8729 & 1.8726 & 1.8705 & 1.8703 & 1.8727 & 1.8730 \\
$\mathrm{k}$ & 15 & 2 & $1.86325(20)$ & 1.8630 & 1.8632 & 1.8610 & 1.8601 & 1.8630 & 1.8631 \\
$\mathrm{l}$ & 15 & 3 & & 1.8677 & 1.8677 & 1.8656 & 1.8652 & 1.8674 & 1.8676 \\
$\mathrm{~d}$ & 16 & 2 & & 1.8626 & 1.8627 & 1.8608 & 1.8594 & 1.8628 & 1.8629 \\
$\mathrm{c}$ & 16 & 3 & & 1.8674 & 1.8672 & 1.8653 & & 1.8672 & 1.8673 \\
$\mathrm{j}$ & 17 & 3 & $1.86576(12)$ & 1.8661 & 1.8658 & 1.8635 & 1.8631 & 1.8659 & 1.8660 \\
$\mathrm{~b}$ & 18 & 2 & & 1.8576 & 1.8579 & 1.8555 & 1.8542 & 1.8578 & 1.8578 \\
$\mathrm{a}$ & 18 & 3 & $1.86207(30)$ & 1.8623 & 1.8624 & 1.8600 & 1.8593 & 1.8622 & 1.8622 \\
$\mathrm{n}$ & 19 & 2 & & 1.8523 & 1.8526 & 1.8526 & 1.8488 & 1.8523 & 1.8521 \\
$\mathrm{~m}$ & 19 & 3 & $1.85693(20)$ & 1.8570 & 1.8570 & 1.8571 & 1.8539 & 1.8566 & 1.8565 \\
\hline & & & & & & & & &
\end{tabular}


Table 4. Comparison of $A$-values $\left(10^{13} \mathrm{~s}^{-1}\right.$ ) for $\mathrm{K}$ transitions in Fe XXIV (see approximation key in Table 1). Note: $a \pm b \equiv a \times 10^{ \pm b}$.

\begin{tabular}{rrllllllllll}
\hline \hline$k$ & $i$ & AST1 & AST2 & AST3 & HFR1 & HFR2 & HFR3 & HFR4 & COR & SAF & MCDF \\
\hline 4 & 2 & $9.76-1$ & $9.46-1$ & $9.27-1$ & $9.62-1$ & $1.03+0$ & $1.00+0$ & & $9.51-1$ & $8.75-1$ & $8.25-1$ \\
4 & 3 & $9.85-1$ & $9.84-1$ & $9.52-1$ & $1.04+0$ & $1.08+0$ & $1.07+0$ & & $9.39-1$ & $9.07-1$ & $8.36-1$ \\
5 & 1 & $4.06-1$ & $4.98-1$ & $4.97-1$ & $3.72-1$ & $4.08-1$ & $2.92-1$ & $4.00-1$ & & $4.92-1$ & $4.86-1$ \\
6 & 1 & $1.40+0$ & $1.55+0$ & $1.55+0$ & $1.26+0$ & $1.40+0$ & $9.60-1$ & $1.50+0$ & $1.47+0$ & $1.59+0$ & $1.54+0$ \\
7 & 1 & $6.18-4$ & $6.18-4$ & $6.16-4$ & & & & & & & \\
7 & 3 & $1.93-5$ & $1.94-5$ & $1.94-5$ & & & & & & & \\
8 & 1 & $2.88+1$ & $3.06+1$ & $3.01+1$ & $3.04+1$ & $3.10+1$ & $3.29+1$ & $2.99+1$ & $2.88+1$ & $3.19+1$ & $2.89+1$ \\
9 & 1 & $4.70+1$ & $4.71+1$ & $4.71+1$ & $4.72+1$ & $4.94+1$ & $4.86+1$ & $4.91+1$ & & $4.87+1$ & $4.43+1$ \\
10 & 2 & $1.90+0$ & $2.02+0$ & $2.17+0$ & $1.72+0$ & $1.89+0$ & $1.88+0$ & $2.90+0$ & & $2.10+0$ & $1.98+0$ \\
10 & 3 & $1.77-2$ & $7.70-3$ & $9.12-3$ & $1.68-2$ & $1.79-2$ & $1.60-2$ & $1.00-1$ & & $9.30-3$ & $1.27-2$ \\
11 & 1 & $2.01+1$ & $1.82+1$ & $1.86+1$ & $1.87+1$ & $2.01+1$ & $1.76+1$ & $2.07+1$ & $2.03+1$ & $1.79+1$ & $1.68+1$ \\
12 & 1 & $8.92-1$ & $5.90-1$ & $4.19-1$ & $1.05+0$ & $6.57-1$ & $1.25+0$ & $5.00-1$ & $4.41-1$ & $7.78-2$ & $3.23-1$ \\
13 & 2 & $6.21-2$ & $6.63-3$ & $4.51-3$ & $7.77-3$ & $9.03-3$ & $1.06-2$ & $0.0+0$ & & $2.40-3$ & $3.42-3$ \\
13 & 3 & $8.01-1$ & $1.01+0$ & $1.06+0$ & $7.54-1$ & $8.11-1$ & $8.13-1$ & $9.00-1$ & $8.23-1$ & $1.01+0$ & $9.67-1$ \\
14 & 3 & $3.11+0$ & $3.11+0$ & $3.58+0$ & $2.81+0$ & $3.10+0$ & $3.21+0$ & $3.40+0$ & $3.37+0$ & $3.51+0$ & $3.17+0$ \\
15 & 2 & $3.13+1$ & $3.17+1$ & $3.14+1$ & $3.14+1$ & $3.26+1$ & $3.24+1$ & $3.16+1$ & $3.15+1$ & $3.27+1$ & $2.96+1$ \\
15 & 3 & $3.39+0$ & $4.32+0$ & $3.64+0$ & $3.37+0$ & $3.49+0$ & $3.26+0$ & $3.50+0$ & $3.09+0$ & $3.90+0$ & $3.80+0$ \\
16 & 2 & $5.39+1$ & $5.35+1$ & $5.31+1$ & $5.40+1$ & $5.62+1$ & $5.53+1$ & $5.58+1$ & $5.39+1$ & $5.44+1$ & $4.97+1$ \\
16 & 3 & $1.58+1$ & $1.63+1$ & $1.60+1$ & $1.62+1$ & $1.66+1$ & $1.65+1$ & $1.29+1$ & & $1.65+1$ & $1.53+1$ \\
17 & 3 & $2.09+1$ & $2.09+1$ & $2.05+1$ & $2.12+1$ & $2.19+1$ & $2.17+1$ & $2.17+1$ & $2.11+1$ & $2.16+1$ & $1.98+1$ \\
18 & 2 & $1.15+0$ & $7.70-1$ & $9.69-1$ & $1.16+0$ & $1.21+0$ & $1.24+0$ & $1.10+0$ & $1.25+0$ & $8.63-1$ & $7.57-1$ \\
18 & 3 & $6.16+1$ & $6.04+1$ & $6.07+1$ & $6.18+1$ & $6.43+1$ & $6.37+1$ & $6.31+1$ & $6.20+1$ & $6.21+1$ & $5.64+1$ \\
19 & 2 & $9.78-1$ & $1.20+0$ & $1.03+0$ & $1.18+0$ & $1.11+0$ & $1.06+0$ & $2.00-1$ & $8.89-1$ & $1.09+0$ & $1.08+0$ \\
19 & 3 & $2.46+1$ & $2.42+1$ & $2.40+1$ & $2.43+1$ & $2.56+1$ & $2.49+1$ & $2.92+1$ & $2.44+1$ & $2.43+1$ & $2.22+1$ \\
\hline & & & & & & & & & & &
\end{tabular}

we hereby demonstrate that in some cases the forbidden transitions cannot be put aside.

In Table 4 we present transition probabilities computed in the different approximations together with those from previous work (HFR4, COR, SAF, and MCDF). In the following discussion, we exclude the transitions $10-3,12-1,13-2$ and 18-2 as they are severely affected by cancellation and nothing further can be asserted about their radiative properties.

In Fig. 1 we compare $A$-values computed in AST2 with those in AST1 where significant differences are found. In general, the inclusion of the Breit interaction (AST2) increases rates; while the variations are not larger than $10 \%$ for the spin allowed transitions that exhibit large rates $\left(\log A_{\mathrm{r}}>14\right)$, the enhancement in the intersystem transitions (5-1, 6-1 and 13-3) can be as large as $25 \%$. Inclusion of CI from the $n=3$ complex leads to changes not larger than $2 \%$, but the fitting with TEC, as expected, causes differences mostly in the sensitive intersystem transitions. By comparing HFR1 and HFR2 (see Table 4), it can be concluded that CRE tend to increase $A$-values but seldom by more than $10 \%$; the exceptions are the transitions affected by strong cancellation (e.g. 12-1 and 13-2).

In Fig. 2a the transition probabilities computed in approximation AST1 are compared with those by HFR2, COR, SAF and MCDF. While there is as expected excellent agreement with COR (within 10\%), the data in HFR2 and SAF are on average higher by $\sim 5 \%$ with scatters of $\pm 4 \%$ and $\pm 12 \%$, respectively. Differences with MCDF are as large as $21 \%$. The discord with HFR2 is due to CRE while that with SAF and MCDF is believed to be due to the contributions of the relativistic twobody corrections excluded in AST1. This assertion is supported

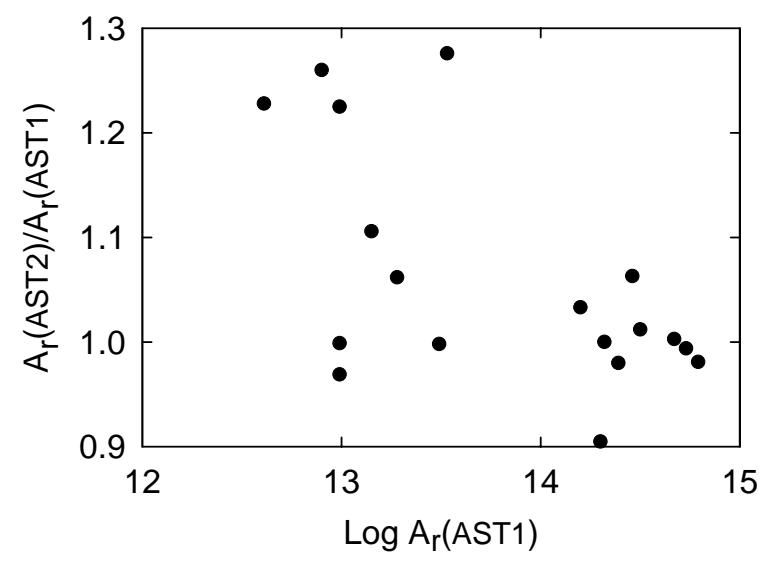

Fig. 1. Comparison of $A$-values $\left(\mathrm{s}^{-1}\right)$ for $\mathrm{K}$ transitions in Fe XXIV computed with approximations AST1 and AST2. Differences are due to the Breit interaction.

by a further comparison with the data in AST3 (Fig. 2b); now the agreement with SAF and MCDF has improved to $\sim 10 \%$ while discrepancies as large as $25 \%$ are found with COR where the Breit interaction was neglected. The larger differences now found with HFR3 (15\%) are an indication that the BlumeWatson screening in HFR does not account adequately for the Breit interaction. The external HFR4 data set is in reasonable agreement (within 20\%) with HFR2 except for the large, unexplainable discrepancies in the 10-2 (53\%) and 19-2 (factor of 5) transitions. The outcome of this comparison give us confidence on the accuracy ranking $(\sim 10 \%)$ that can be 
Table 5. $A$-values $\left(10^{9} \mathrm{~s}^{-1}\right)$ for $\mathrm{K}$ transitions with sizable magnetic components computed in approximation AST3. E1: electric dipole. M2: magnetic quadrupole. M1: magnetic dipole. M1*: magnetic dipole computed with the uncorrected operator. Note: $a \pm b \equiv a \times 10^{ \pm b}$.

\begin{tabular}{rrllll}
\hline \hline$k$ & $i$ & E1 & M2 & M1 & M1* \\
\hline 7 & 1 & 0.0 & $6.16+0$ & 0.0 & \\
7 & 3 & 0.0 & 0.0 & $1.94-1$ & $6.11-7$ \\
10 & 3 & $9.07+1$ & $5.04-1$ & 0.0 & \\
13 & 2 & $3.99+1$ & $5.19+0$ & 0.0 & \\
\hline
\end{tabular}

assigned to the $A$-values in AST3 which we regard our best approximation.

We have found that the K-vacancy states in Li-like iron, in addition to their dipole allowed manifold, can also decay radiatively via unusually strong magnetic transitions. As shown in Table 5, the $A$-values for the M2 components in 10-3 and 13-2 are almost as large as their E1 counterparts, and therefore must be taken into account in order to maintain accuracy. The situation becomes critical for the $1 \mathrm{~s} 2 \mathrm{~s} 2 \mathrm{p}{ }^{4} \mathrm{P}_{5 / 2}^{0}$ metastable which is shown to decay through both M1 and M2 transitions (see Table 5). It may be also appreciated that the M1 $A$-value must be calculated with the relativistically corrected operator (see Eq. (9)) since the difference with the uncorrected version is 5 orders of magnitude. Chen et al. (1981) have assumed that this state decays radiatively only via the M2 transition, and quote a value of $A_{\mathrm{r}}=6.57 \times 10^{9} \mathrm{~s}^{-1}$ in good agreement $(7 \%)$ with the present $A_{\mathrm{M} 2}=6.16 \times 10^{9} \mathrm{~s}^{-1}$.

\section{Auger rates}

While the radiative transition probabilities can be resolved satisfactorily, the effects of magnetic couplings and CRE on the Auger rates are more evident and thus larger the discrepancies. A Li-like $1 s 2 l 2 l^{\prime}$ level autoionizes through the reaction

$1 \mathrm{~s} 2 \mathrm{~s}^{n_{k}} 2 \mathrm{p}^{m_{k}\left(2 S_{k}+1\right)} L_{J_{k}} \rightarrow 1 \mathrm{~s}^{2}{ }^{2} \mathrm{~S}_{0}+\mathrm{e}^{-}$

that ends up in the ground state of the He-like child ion. A comparison of rates is given in Table 6. As before, due to strong cancellation effects, we exclude the $1 \mathrm{~s}\left({ }^{2} \mathrm{~S}\right) 2 \mathrm{~s} 2 \mathrm{p}\left({ }^{3} \mathrm{P}^{0}\right){ }^{2} \mathrm{P}_{3 / 2}^{0}$ and ${ }^{4} \mathrm{P}_{1 / 2}^{0}$ states from further discussion. By comparing data from approximations AST1 and AST2 (see Fig. 3), it is found significant sensitivity to the Breit interaction: states with $\log A_{\mathrm{a}}>13$ are in general reduced by no more than $11 \%$, but the smaller values show decrements as large as a factor of 2 . As shown in Table 7, the spin-spin interaction can cause drastic changes in the rates, not only due to level coupling within the parent bound configurations (bound-bound coupling) but also involving the final continuum configuration (bound-free coupling). An outstanding illustration of this correlation is the $1 \mathrm{~s} 2 \mathrm{~s} 2 \mathrm{p}^{4} \mathrm{P}_{5 / 2}^{0}$ state which can only autoionize through the spinspin interaction. By contrast, CI from the $n=3$ complex is found to be relatively unimportant, but the TEC lead to noticeable changes $(25 \%)$ in the quartet states, e.g. $1 \mathrm{~s} 2 \mathrm{p}^{2}{ }^{4} \mathrm{P}_{J}$, that can only decay through relativistic intersystem couplings that are sensitive to level separation. The good agreement $(\sim 10 \%)$ between AST1 and BPR1 for states with $\log A_{\mathrm{a}}>13$ (see Table 6)
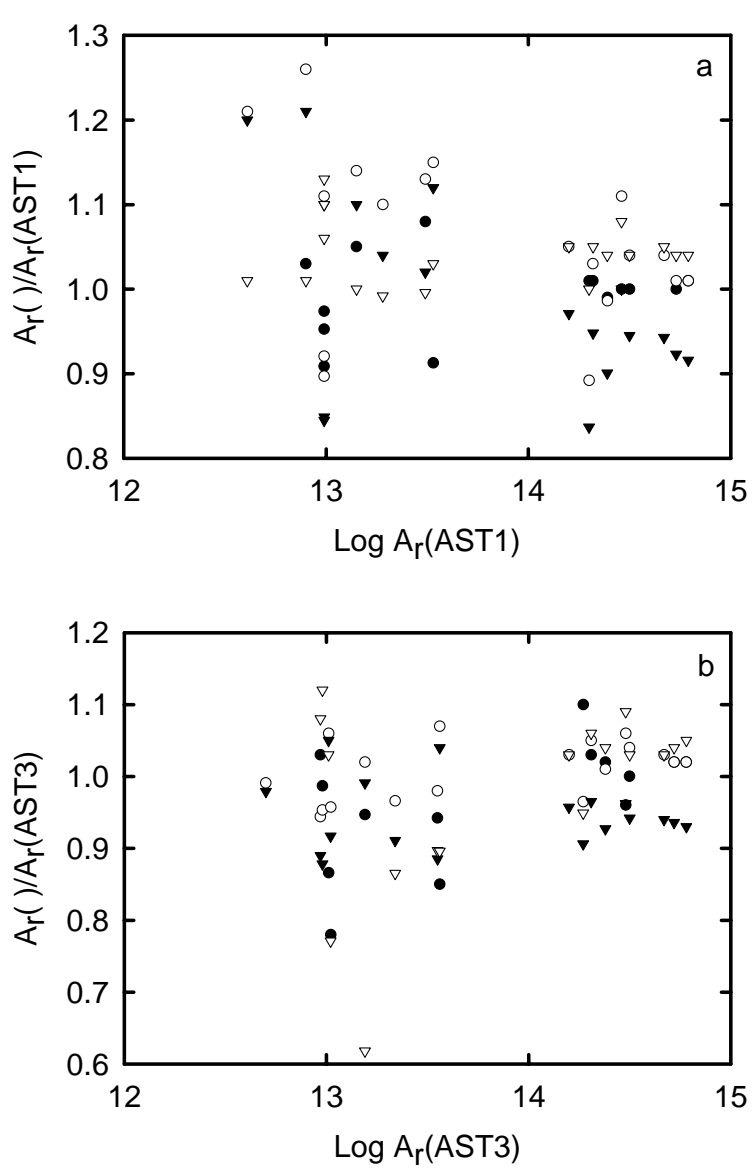

Fig. 2. Comparison of AUTOSTRUCtURE $A$-values $\left(\mathrm{s}^{-1}\right)$ for $\mathrm{K}$ transitions in Fe XXIV with other approximations and external data sets. a) AST1 with: HFR2 (triangles); COR (filled circles); SAF (circles); and MCDF (filled triangles). b) AST3 with: HFR3 (triangles); COR (filled circles); SAF (circles); and MCDF (filled triangles).

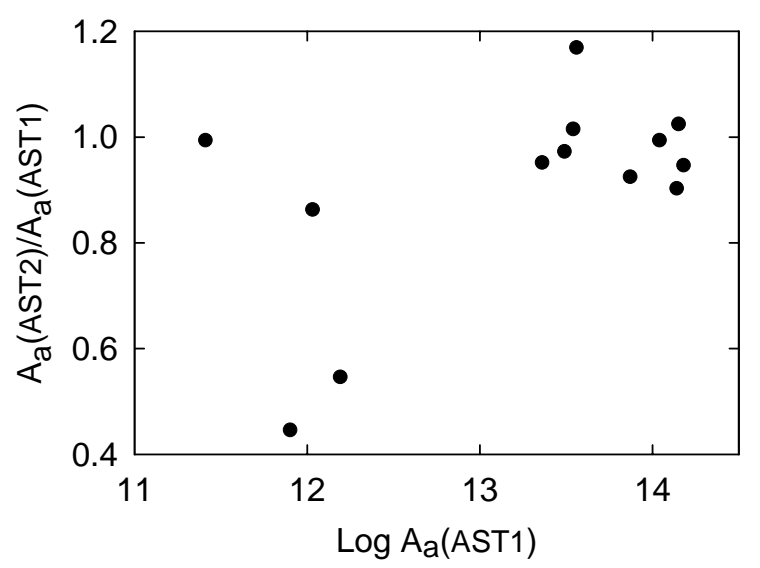

Fig. 3. Comparison of Auger rates $\left(\mathrm{s}^{-1}\right)$ for K-vacancy levels of Fe XXIV computed with approximations AST1 and AST2. Differences are due to the Breit interaction.

reinforces the AUTOSTRUCTURE numerical formulation of autoionization processes. CRE in Auger decay are disclosed in the comparison of HFR1 and HFR2 where it is found that relaxation generally increases widths by $(11 \pm 5) \%$. Discrepancies between AST2 and HFR2 and AST3 and HFR3, which can be as large as $45 \%$ for transitions with $\log A_{\mathrm{a}}>13$, are believed 
Table 6. Comparison of Auger rates $\left(10^{13} \mathrm{~s}^{-1}\right.$ ) for K-vacancy states in Fe XXIV (see approximation key in Table 1). Note: $a \pm b \equiv a \times 10^{ \pm b}$.

\begin{tabular}{rllllllllll}
\hline \hline$i$ & AST1 & AST2 & AST3 & HFR1 & HFR2 & HFR3 & BPR1 & COR & SAF & MCDF \\
\hline 4 & $1.40+1$ & $1.44+1$ & $1.43+1$ & $1.25+1$ & $1.34+1$ & $1.34+1$ & $1.45+1$ & $1.41+1$ & $1.47+1$ & $1.42+1$ \\
5 & $1.88-2$ & $1.45-3$ & $1.33-3$ & $1.36-2$ & $1.54-2$ & $1.09-2$ & $1.57-2$ & & $1.19-2$ & $5.57-3$ \\
6 & $7.96-2$ & $3.55-2$ & $3.91-2$ & $5.74-2$ & $6.56-2$ & $4.31-2$ & $7.07-2$ & $8.40-2$ & $8.85-2$ & $1.71-2$ \\
7 & $0.00+0$ & $1.99-4$ & $1.97-4$ & $0.00+0$ & $0.00+0$ & $0.00+0$ & $0.00+0$ & & & \\
8 & $3.67+0$ & $4.29+0$ & $4.24+0$ & $2.94+0$ & $3.42+0$ & $2.92+0$ & $3.87+0$ & $3.80+0$ & $3.21+0$ & $4.83+0$ \\
9 & $8.99-4$ & $2.34-2$ & $1.41-2$ & $5.01-2$ & $3.01-2$ & $8.57-2$ & $1.55-2$ & & $3.02-2$ & $5.74-2$ \\
10 & $2.55-2$ & $2.53-2$ & $3.37-2$ & $1.58-2$ & $1.94-2$ & $2.25-2$ & $3.15-2$ & & $3.24-2$ & $1.53-2$ \\
11 & $7.43+0$ & $6.87+0$ & $6.77+0$ & $6.91+0$ & $7.16+0$ & $7.55+0$ & $7.74+0$ & $7.40+0$ & $8.96+0$ & $7.00+0$ \\
12 & $1.10+1$ & $1.10+1$ & $1.07+1$ & $9.77+0$ & $1.05+1$ & $1.04+1$ & $1.11+1$ & $1.10+1$ & $1.21+1$ & $1.05+1$ \\
13 & $1.55-1$ & $8.44-2$ & $9.66-2$ & $1.18-1$ & $1.37-1$ & $1.41-1$ & $1.78-1$ & $1.58-1$ & $1.01-1$ & $4.30-2$ \\
14 & $2.31+0$ & $2.20+0$ & $2.61+0$ & $1.75+0$ & $2.05+0$ & $2.12+0$ & $2.56+0$ & $2.36+0$ & $2.64+0$ & $2.17+0$ \\
15 & $1.39+1$ & $1.26+1$ & $1.25+1$ & $1.17+1$ & $1.29+1$ & $1.30+1$ & $1.38+1$ & $1.35+1$ & $1.44+1$ & $1.27+1$ \\
16 & $1.06-1$ & $9.16-2$ & $9.39-2$ & $6.60-2$ & $8.17-2$ & $8.11-2$ & $7.01-2$ & $9.50-2$ & $9.08-2$ & $1.64-1$ \\
17 & $1.52+1$ & $1.44+1$ & $1.37+1$ & $1.31+1$ & $1.44+1$ & $1.43+1$ & $1.47+1$ & $1.46+1$ & $1.60+1$ & $1.42+1$ \\
18 & $3.44+0$ & $3.49+0$ & $3.28+0$ & $3.05+0$ & $3.37+0$ & $3.27+0$ & $3.19+0$ & $3.29+0$ & $4.16+0$ & $3.14+0$ \\
19 & $3.09+0$ & $3.00+0$ & $2.92+0$ & $2.40+0$ & $2.77+0$ & $2.76+0$ & $2.75+0$ & $2.83+0$ & $3.21+0$ & $2.72+0$ \\
\hline
\end{tabular}

to be due to both CRE and the oversimplified implementation of the Breit interaction in HFR.

In Fig. 4 Auger rates in AST1 and AST3 are compared with COR, SAF, and MCDF. While agreement between COR and AST1 is within $10 \%$, it clearly deteriorates with AST3; this is further evidence of the neglect of the Breit interaction by COR. Significant differences are also found with SAF and MCDF in particular for the smaller values $\left(\log A_{\mathrm{a}}<13\right)$. Focusing our discussion on the larger rates, data by SAF are on average $8 \%$ higher than AST1 (see Fig. 4a) which is a worrying outcome because the inclusion of the Breit interaction in general decreases our rates thus magnifying the discrepancy. This can be appreciated in the comparison of SAF with AST3 in Fig. 4b where the larger differences are found for decays subject to strong spin-spin bound-free correlation (see Table 7), and can perhaps be attributed to its deficient treatment in the SAF approach. By contrast, the discord between AST1 and MCDF for the larger rates (up to $32 \%$ ) is reduced to within $15 \%$ when the Breit interaction is taken into account.

The lack of data stability for Auger transitions with $\log A_{\mathrm{a}}<13$ is further put in evidence in the tricky decay of the $1 \mathrm{~s} 2 \mathrm{~s} 2 \mathrm{p}{ }^{4} \mathrm{P}_{5 / 2}^{0}$ state. While there is good agreement with Chen et al. (1981) for the dominant radiative M2 A-value (see Sect. 7), their Auger rate of $6.53 \times 10^{9} \mathrm{~s}^{-1}$ is a factor of 3 larger thus predicting a lower fluorescence yield (0.50) than the present $(0.76)$ for this state.

\section{9. $B_{\mathrm{r}}$ and $Q_{\mathrm{d}}$ factors}

In the spectral synthesis of dielectronic satellite lines, relevant parameters for a $k \rightarrow i$ radiative emission are the branching ratio

$B_{\mathrm{r}}(k, i) \equiv \frac{A_{\mathrm{r}}(k, i)}{A_{\mathrm{r}}(k)+A_{\mathrm{a}}(k)}$

and the satellite intensity factor

$Q_{\mathrm{d}}(k, i) \equiv g_{k} B_{\mathrm{r}}(k, i) A_{\mathrm{a}}(k)$
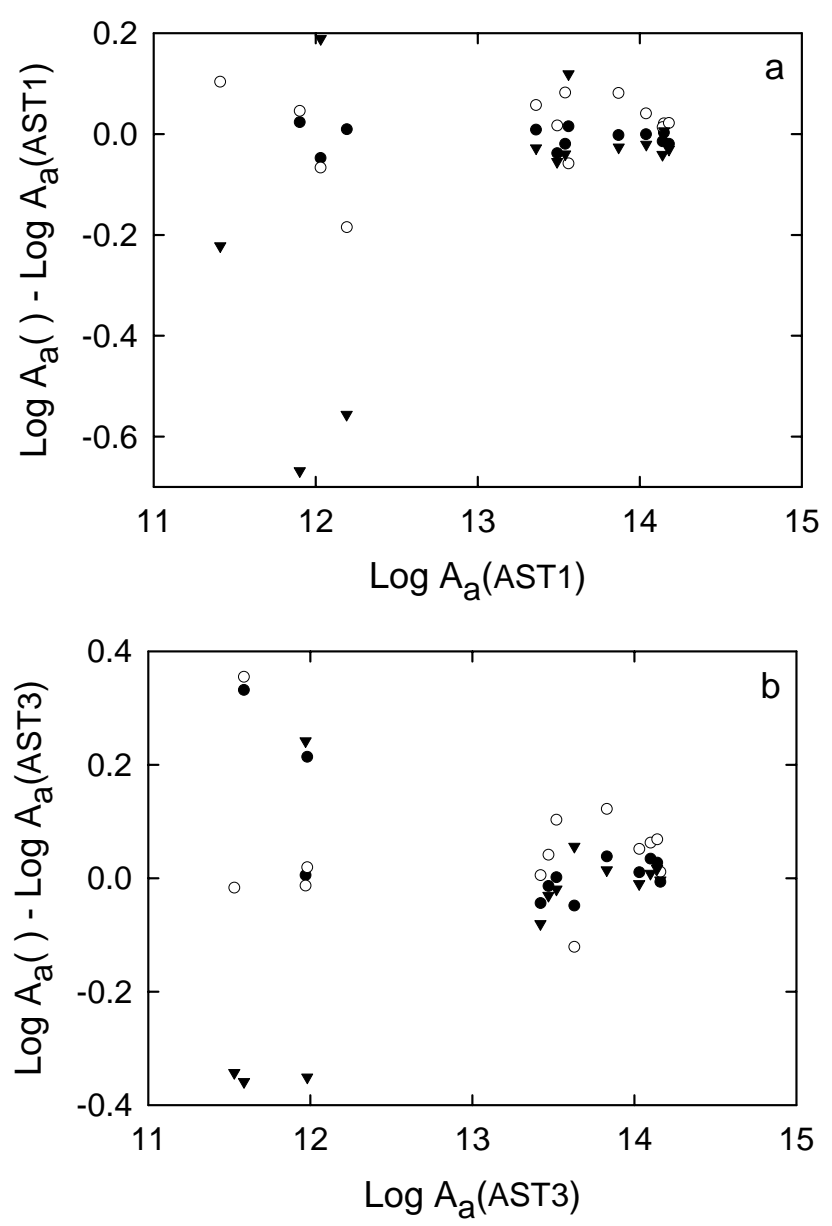

Fig. 4. Comparison of AUTOSTRUCTURE Auger rates $\left(\mathrm{s}^{-1}\right)$ for Kvacancy levels in Fe XXIV with previous data sets. a) AST1 with: COR (filled circles); SAF (circles); and MCDF (filled triangles). b) AST3 with: COR (filled circles); SAF (circles); and MCDF (filled triangles).

where $A_{\mathrm{r}}(k, i), A_{\mathrm{r}}(k)=\sum_{i} A_{\mathrm{r}}(k, i), A_{\mathrm{a}}(k)$ and $g_{k}$ are respectively the $A$-value, total radiative width, Auger rate and 
Table 7. Spin-spin contribution to Auger rates $\left(10^{13} \mathrm{~s}^{-1}\right)$. SS: boundfree spin-spin coupling neglected. SS*: bound-free spin-spin coupling included. Note: $a \pm b \equiv a \times 10^{ \pm b}$.

\begin{tabular}{rlll}
\hline \hline$i$ & AST1 & AST1+SS & AST1+SS* \\
\hline 4 & $1.40+1$ & $1.31+1$ & $1.40+1$ \\
5 & $1.88-2$ & $3.70-3$ & $3.42-3$ \\
6 & $7.96-2$ & $4.27-2$ & $2.96-2$ \\
7 & 0.0 & 0.0 & $1.99-4$ \\
8 & $3.67+0$ & $3.92+0$ & $3.98+0$ \\
9 & $8.99-4$ & $1.61-1$ & $4.24-3$ \\
10 & $2.55-2$ & $2.11-2$ & $2.69-2$ \\
11 & $7.43+0$ & $6.47+0$ & $7.52+0$ \\
12 & $1.10+1$ & $1.02+1$ & $1.09+1$ \\
13 & $1.55-1$ & $7.99-2$ & $3.82-2$ \\
14 & $2.31+0$ & $2.00+0$ & $2.06+0$ \\
15 & $1.39+1$ & $1.14+1$ & $1.41+1$ \\
16 & $1.06-1$ & $7.37-2$ & $1.01-1$ \\
17 & $1.52+1$ & $1.29+1$ & $1.57+1$ \\
18 & $3.44+0$ & $3.42+0$ & $3.11+0$ \\
19 & $3.09+0$ & $2.67+0$ & $3.09+0$ \\
\hline
\end{tabular}

statistical weight of the upper $k$ level. In Table 8 we compare our best data set (AST3) with HFR4, COR, SAF and MCDF. For $B_{\mathrm{r}}>0.1$, the agreement is within $5 \%$ except for the COR 13-3 and the SAF 11-1 lines where it deteriorates to $9 \%$, and for the 10-2 (13\%) and 16-3 (19\%) lines in HFR4. The 13-3 transition, being an intercombination transition, is sensitive to the atomic model while level 11 is subject to admixture. For $B_{\mathrm{r}}<0.1$, the accord is within $15 \%$ if transitions affected with cancellation are put aside and the 19-2 transition in HFR4 is excluded. Furthermore, for $Q_{\mathrm{d}}>10^{13} \mathrm{~s}^{-1}$, the agreement with HFR4, COR, SAF and MCDF is respectively within $20 \%$, $10 \%, 25 \%$ and $15 \%$ except for the 19-3 transition in HFR4 where an anomalous discrepancy of almost a factor of 2 is encountered. For the smaller values, discrepancies up to a factor of 9 do appear.

Beiersdorfer et al. (1992) have measured the strength of dielectronic satellite lines in Li-like Fe excited by an electronic beam which can be compared with the theoretical values given by the relation

$S_{\mathrm{d}}(k, i)=\frac{Q_{\mathrm{d}}(k, i)}{g_{I}} \frac{1}{E_{k}} 2 \pi^{2} a_{0}^{3} R^{2}\left(\frac{m_{\mathrm{e}}}{2 R}\right)^{1 / 2}$

where $E_{k}$ is the resonance energy, $g_{I}$ is the statistical weight of the ground state of the recombining ion, and $R$ and $a_{0}$ are the Rydberg constant and Bohr radius, respectively. In Table 9 the ratios of the theoretical values to the measurements as defined in Beiersdorfer et al. (1992) are tabulated. On average the AST3, COR, SAF and MCDF ratios are within the experimental uncertainty of $20 \%$, but MCDF shows the best agreement $(10 \%)$. Overall, this comparison seems to suggest that the experimental strengths should perhaps be scaled down by 5$10 \%$. It can also be seen that the strength for the m line obtained in HFR4 is a factor of 2 larger than experiment.

\section{Electron impact inner-shell excitation of Fe XXIV}

Inner-shell vacancy states in Fe XXIV can also be poputaled by electron impact excitation

$1 \mathrm{~s}^{2} 2 l_{i}^{2} L_{J_{i}}+\mathrm{e}^{-} \rightarrow 1 \mathrm{~s} 2 \mathrm{~s}^{n_{k}} 2 \mathrm{p}^{m_{k}\left(2 S_{k}+1\right)} L_{J_{k}}^{\prime}+\mathrm{e}^{-}$.

As summarized by Pradhan \& Gallagher (1992), collision strengths for this reaction have been computed with a variety of methods; for instance, distorted-wave (Bely-Dubau et al. 1982) and Coulomb-Born-Exchange (Goett et al. 1984; Sampson et al. 1985). More recently, BPRM has been used by Ballance et al. (2001) and Whiteford et al. (2002) using target representations that include doubly excited levels up to the $n=3$ shell. In the present calculation, BPRM is used with a target representation that includes only the 19 levels within the $n=2$ complex since exploratory calculations with $n=3$ target states lead to small differences in the energy region of interest. We are particularly concerned with the effects of radiative and Auger dampings and the convergence of the partial wave expansion.

In Fig. 5 collision strengths for both an allowed (1-8) and a forbidden (1-14) transition are shown. Although the background cross section is generally small $(\log \Omega<-2)$, specially for the latter transition type, they both display dense resonance structures in the region just above threshold that rise by several orders of magnitude. When radiation damping is introduced, however, resonances are washed out in the allowed transition and significantly attenuated in the forbidden case, trend that is further completed when Auger damping is taken into account. In agreement with Whiteford et al. (2002), the effect of the combined dampings on the low-temperature effective collision strengths can be drastic as illustrated in Table 10 where differences of factors are seen. The extreme case is the forbidden transition 1-13 that is overestimated by nearly two orders of magnitude if damping is altogether neglected and by a factor of two with the exclusion of Auger damping. It must be pointed out that the calculation by Ballance et al. (2001) of inner-shell excitation of $\mathrm{Li}$ - and $\mathrm{Be}$-like $\mathrm{Fe}$ does not take into account Auger damping.

With regards to relativistic effects, the collision strengths for the fine structure transitions have been calculated in three different approximations: (a) $L S$-coupling followed by algebraic recoupling; (b) $L S$-coupling followed by recoupling with term coupling coefficients that account for target fine structure and (c) the relativistic Hamiltonian (Eq. (2)) that includes only the one-body operators. Good agreement is found between approximations (b) and (c) while large discrepancies are found with (a). These results indicate that relativistic effects must be taken into account in the scattering formulation and that the two-body corrections, which are not implemented in BPRM, are small and can be neglected in this case.

Under coronal ionization conditions the temperatures of maximum abundance of Fe XXIII and Fe XXIV are $\sim 2 \times 10^{7} \mathrm{~K}$ and $\sim 4 \times 10^{7} \mathrm{~K}$ respectively; effective collision strengths must be then computed at temperatures of up to $10^{8} \mathrm{~K}$. To ensure accuracy in the Maxwellian averaging integral, collision strengths are computed in a range up to 4000 Ryd where partial 
Table 8. Comparison of radiative branching ratios $B_{\mathrm{r}}$ and satellite intensity $Q_{\mathrm{d}}$ factors $\left(10^{13} \mathrm{~s}^{-1}\right)$. Approximation key is given in Table 1 . Note: $a \pm b \equiv a \times 10^{ \pm b}$.

\begin{tabular}{|c|c|c|c|c|c|c|c|c|c|c|c|}
\hline \multirow[b]{2}{*}{$k$} & \multirow[b]{2}{*}{$i$} & \multicolumn{5}{|c|}{$B_{\mathrm{r}}(k, i)$} & \multicolumn{5}{|c|}{$Q_{\mathrm{d}}(k, i)$} \\
\hline & & AST3 & HFR4 & COR & SAF & MCDF & AST3 & HFR4 & COR & SAF & MCDF \\
\hline 4 & 2 & $5.72-2$ & & $6.00-2$ & $5.29-2$ & $5.20-2$ & $1.64+0$ & & $1.68+0$ & $1.56+0$ & $1.48+0$ \\
\hline 4 & 3 & $5.88-2$ & & $5.90-2$ & $5.49-2$ & $5.25-2$ & $1.68+0$ & & $1.66+0$ & $1.62+0$ & $1.50+0$ \\
\hline 5 & 1 & $9.97-1$ & $9.65-1$ & & $9.76-1$ & $9.90-1$ & $2.66-3$ & $0.00+0$ & & $2.32-2$ & $1.10-2$ \\
\hline 6 & 1 & $9.75-1$ & $9.57-1$ & $9.46-1$ & $9.47-1$ & $9.90-1$ & $1.53-1$ & $3.00-1$ & $3.17-1$ & $3.35-1$ & $6.78-2$ \\
\hline 7 & 1 & $7.40-1$ & & & & & $8.76-4$ & & & & \\
\hline 7 & 3 & $2.32-2$ & & & & & $2.75-5$ & & & & \\
\hline 8 & 1 & $8.76-1$ & $9.01-1$ & $8.83-1$ & $9.09-1$ & $8.55-1$ & $7.44+0$ & $5.90+0$ & $6.72+0$ & $5.83+0$ & $8.28+0$ \\
\hline 9 & 1 & $1.00+0$ & $1.00+0$ & & $9.99-1$ & $9.98-1$ & $5.64-2$ & $0.00+0$ & & $1.21-1$ & $2.29-1$ \\
\hline 10 & 2 & $9.81-1$ & $8.54-1$ & & $9.81-1$ & $9.85-1$ & $6.61-2$ & $7.00-1$ & & $6.35-2$ & $3.01-2$ \\
\hline 10 & 3 & $4.12-3$ & $3.20-2$ & & $4.35-3$ & $6.30-3$ & $2.77-4$ & $0.00+0$ & & $2.82-4$ & $1.93-4$ \\
\hline 11 & 1 & $7.33-1$ & $7.60-1$ & $7.33-1$ & $6.67-1$ & $7.05-1$ & $9.92+0$ & $9.90+0$ & $1.08+1$ & $1.19+1$ & $9.88+0$ \\
\hline 12 & 1 & $3.76-2$ & $4.70-2$ & $3.80-2$ & $6.41-3$ & $3.00-2$ & $1.61+0$ & $1.80+0$ & $1.70+0$ & $3.09-1$ & $1.25+0$ \\
\hline 13 & 2 & $3.90-3$ & $1.10-2$ & & $2.16-3$ & $3.38-3$ & $1.51-3$ & $0.00+0$ & & $8.72-4$ & $5.81-4$ \\
\hline 13 & 3 & $9.13-1$ & $8.55-1$ & $8.27-1$ & $9.07-1$ & $9.53-1$ & $3.53-1$ & $5.00-1$ & $5.23-1$ & $3.67-1$ & $1.64-1$ \\
\hline 14 & 3 & $5.78-1$ & $6.26-1$ & $5.88-1$ & $5.71-1$ & $5.93-1$ & $9.06+0$ & $7.70+0$ & $8.34+0$ & $9.04+0$ & $7.72+0$ \\
\hline 15 & 2 & $6.61-1$ & $6.83-1$ & $6.55-1$ & $6.41-1$ & $6.43-1$ & $3.29+1$ & $3.18+1$ & $3.53+1$ & $3.70+1$ & $3.25+1$ \\
\hline 15 & 3 & $7.66-2$ & $7.40-2$ & $6.40-2$ & 7.64-2 & $8.25-2$ & $3.82+0$ & $3.40+0$ & $3.47+0$ & $4.41+0$ & $4.18+0$ \\
\hline 16 & 2 & $7.68-1$ & $8.07-1$ & $7.72-1$ & $7.67-1$ & $7.65-1$ & $1.44-1$ & $7.00-1$ & $1.47-1$ & $1.39-1$ & $2.51-1$ \\
\hline 16 & 3 & $2.31-1$ & $1.87-1$ & & $2.32-1$ & $2.35-1$ & $4.34-2$ & $2.00-1$ & & $4.21-2$ & $7.70-2$ \\
\hline 17 & 3 & $6.00-1$ & $6.29-1$ & $5.92-1$ & $5.73-1$ & $5.83-1$ & $4.92+1$ & $4.84+1$ & $5.17+1$ & $5.52+1$ & $4.95+1$ \\
\hline 18 & 2 & $1.49-2$ & $1.60-2$ & $1.90-2$ & $1.29-2$ & $1.26-2$ & $1.96-1$ & $2.00-1$ & $2.47-1$ & $2.14-1$ & $1.58-1$ \\
\hline 18 & 3 & $9.35-1$ & $9.38-1$ & $9.31-1$ & $9.25-1$ & $9.35-1$ & $1.23+1$ & $1.17+1$ & $1.23+1$ & $1.54+1$ & $1.18+1$ \\
\hline 19 & 2 & $3.68-2$ & $6.00-3$ & $3.20-2$ & $3.82-2$ & $4.16-2$ & $2.15-1$ & $1.00-1$ & $1.79-1$ & $2.45-1$ & $2.26-1$ \\
\hline 19 & 3 & $8.59-1$ & $8.41-1$ & $8.67-1$ & $8.50-1$ & $8.55-1$ & $5.01+0$ & $8.90+0$ & $4.90+0$ & $5.46+0$ & $4.64+0$ \\
\hline
\end{tabular}

Table 9. Comparison of the dielectronic resonance strengths measured by Beiersdorfer et al. (1992) with the theoretical values. The quantities listed are the ratios of the theoretical values to the measurements as defined in Beiersdorfer et al. (1992). Approximation key is given in Table 1, and the transition labels are from Gabriel (1972).

\begin{tabular}{llllll}
\hline \hline Label & AST3 & HFR4 & COR & SAF & MCDF \\
\hline $\mathrm{e}$ & 1.24 & 1.05 & 1.14 & 1.24 & 1.06 \\
$\mathrm{j}(+\mathrm{l})$ & 1.02 & 1.00 & 1.07 & 1.15 & 1.03 \\
$\mathrm{k}(+\mathrm{a})$ & 0.87 & 0.84 & 0.93 & 0.98 & 0.86 \\
$\mathrm{~m}$ & 1.18 & 2.10 & 1.15 & 1.29 & 1.10 \\
$\mathrm{r}$ & 0.80 & 0.63 & 0.72 & 0.62 & 0.89 \\
$\mathrm{t}(+\mathrm{s})$ & 1.22 & 1.25 & 1.32 & 1.24 & 1.17 \\
$\mathrm{o}$ & 1.29 & & 1.28 & 1.25 & 1.16 \\
$\mathrm{p}$ & 1.21 & & 1.24 & 1.15 & 1.10 \\
\hline
\end{tabular}

wave convergence becomes the main issue. The calculation is performed in two stages: a full BPRM calculation for total angular momentum of the $(N+1)$-electron system in the range $0 \leq J \leq 10$ and a non-exchange calculation for higher $J$ which is carried out in $L S$ coupling and then recoupled with term coupling coefficients. Very good agreement is found in the high-energy region with the Coulomb-Born-Exchange collision strengths by Goett et al. (1984).

Maxwellian averaged collision strengths are listed in Table 11 for the electron-temperature range $5 \leq \log T \leq 8$ for all the $n=2 \mathrm{~K}$ transitions. Infinite-temperature limits are also included. In order to compare with previous work, the data sets are scaled with the techniques developed by Burgess \& Tully (1992). The effective collision strength $\Upsilon(T)$ is mapped onto
Table 10. Effective collision strengths at $3.0 \times 10^{5} \mathrm{~K}$ for transitions from the $1 \mathrm{~s}^{2} 2 \mathrm{~s}^{2} \mathrm{~S}_{1 / 2}$ ground level to the K-vacancy levels of Fe XXIV showing the effects of radiation and Auger dampings. ND: computed without damping. $\mathrm{RD}$ : radiation damping is included. $\mathrm{R}+\mathrm{AD}$ : radiation and Auger dampings are included. Note: $a \pm b \equiv a \times 10^{ \pm b}$.

\begin{tabular}{rrlll}
\hline \hline$i$ & $k$ & $\mathrm{ND}$ & $\mathrm{RD}$ & $\mathrm{R}+\mathrm{AD}$ \\
\hline 1 & 4 & $2.96-3$ & $1.19-3$ & $1.11-3$ \\
1 & 5 & $1.26-3$ & $5.43-4$ & $5.05-4$ \\
1 & 6 & $2.23-3$ & $1.55-3$ & $1.19-3$ \\
1 & 9 & $3.19-3$ & $2.94-3$ & $2.92-3$ \\
1 & 10 & $3.28-5$ & $1.60-5$ & $1.49-5$ \\
1 & 13 & $6.36-5$ & $4.37-6$ & $2.05-6$ \\
1 & 14 & $1.70-5$ & $6.41-6$ & $2.61-6$ \\
1 & 15 & $4.07-6$ & $3.71-6$ & $1.46-6$ \\
1 & 17 & $6.54-6$ & $6.11-6$ & $1.98-6$ \\
1 & 18 & $3.34-6$ & $3.28-6$ & $2.08-6$ \\
\hline
\end{tabular}

the reduced form $\Upsilon_{\mathrm{r}}\left(T_{\mathrm{r}}\right)$ where the infinite temperature $T$ range is scaled to the finite interval $0 \leq T_{\mathrm{r}} \leq 1$. For an allowed transition the scaling is given by the relations

$$
\begin{aligned}
& T_{\mathrm{r}}=1-\frac{\ln (c)}{\ln (\kappa T / \Delta E+c)} \\
& \Upsilon_{\mathrm{r}}\left(T_{\mathrm{r}}\right)=\frac{\Upsilon(T)}{\ln (\kappa T / \Delta E+\mathrm{e})}
\end{aligned}
$$

with $\Delta E$ being the transition energy, $\kappa$ the Boltzmann constant and $c$ an adjustable scaling parameter. For an electric dipole transition the important limit points are

$\Upsilon_{\mathrm{r}}(0)=\Omega(0)$ 
Table 11. Electron impact effective collision strengths for $\mathrm{K}$ transitions within the $n=2$ complex of Fe XXIV.

\begin{tabular}{|c|c|c|c|c|c|c|c|c|c|}
\hline \multicolumn{10}{|c|}{ Electron Temperature (K) } \\
\hline$i$ & $k$ & $1.00+5$ & $5.00+5$ & $1.00+6$ & $5.00+6$ & $1.00+7$ & $5.00+7$ & $1.00+8$ & $\infty$ \\
\hline 1 & 4 & $1.13-3$ & $1.09-3$ & $1.06-3$ & $1.03-3$ & $1.03-3$ & $1.04-3$ & $1.06-3$ & $1.16-3$ \\
\hline 1 & 5 & $5.18-4$ & $4.98-4$ & $4.88-4$ & $4.52-4$ & $4.19-4$ & $2.89-4$ & $2.23-4$ & $3.50-5$ \\
\hline 1 & 6 & $1.31-3$ & $1.13-3$ & $1.06-3$ & $9.62-4$ & $9.01-4$ & $6.89-4$ & $6.02-4$ & $2.41-4$ \\
\hline 1 & 7 & $1.42-3$ & $1.40-3$ & $1.39-3$ & $1.31-3$ & $1.21-3$ & $7.90-4$ & $5.69-4$ & 0.0 \\
\hline 1 & 8 & $1.08-3$ & $1.08-3$ & $1.09-3$ & $1.16-3$ & $1.23-3$ & $1.82-3$ & $2.24-3$ & $2.44-3$ \\
\hline 1 & 9 & $2.92-3$ & $2.94-3$ & $2.99-3$ & $3.26-3$ & $3.55-3$ & $5.66-3$ & $7.25-3$ & $7.93-3$ \\
\hline 1 & 10 & $1.51-5$ & $1.48-5$ & $1.47-5$ & $1.47-5$ & $1.46-5$ & $1.45-5$ & $1.46-5$ & $1.52-5$ \\
\hline 1 & 11 & $8.87-4$ & $8.89-4$ & $8.96-4$ & $9.37-4$ & $9.82-4$ & $1.39-3$ & $1.86-3$ & $1.68-3$ \\
\hline 1 & 12 & $9.53-4$ & $9.39-4$ & $9.32-4$ & $8.84-4$ & $8.25-4$ & $6.49-4$ & $8.00-4$ & $1.49-4$ \\
\hline 1 & 13 & $2.68-6$ & $1.84-6$ & $1.61-6$ & $1.18-6$ & $9.48-7$ & $4.11-7$ & $2.50-7$ & $9.89-10$ \\
\hline 1 & 14 & $2.79-6$ & $2.44-6$ & $2.21-6$ & $1.68-6$ & $1.38-6$ & $6.64-7$ & $4.46-7$ & $2.24-8$ \\
\hline 1 & 15 & $1.54-6$ & $1.41-6$ & $1.35-6$ & $1.20-6$ & $1.10-6$ & $8.87-7$ & $8.03-7$ & \\
\hline 1 & 16 & $7.42-6$ & $7.30-6$ & $7.29-6$ & $7.24-6$ & $7.13-6$ & $6.93-6$ & $6.89-6$ & $6.49-6$ \\
\hline 1 & 17 & $2.24-6$ & $1.87-6$ & $1.75-6$ & $1.50-6$ & $1.36-6$ & $1.06-6$ & $9.59-7$ & \\
\hline 1 & 18 & $2.14-6$ & $2.05-6$ & $2.01-6$ & $1.81-6$ & $1.64-6$ & $1.25-6$ & $1.09-6$ & $2.32-8$ \\
\hline 1 & 19 & $6.61-5$ & $6.59-5$ & $6.64-5$ & $6.69-5$ & $6.65-5$ & $6.64-5$ & $6.69-5$ & \\
\hline 2 & 4 & $1.05-4$ & $9.45-5$ & $8.49-5$ & $7.19-5$ & $7.04-5$ & $8.04-5$ & $9.05-5$ & $8.66-5$ \\
\hline 2 & 5 & $2.98-4$ & $2.90-4$ & $2.73-4$ & $2.38-4$ & $2.21-4$ & $1.57-4$ & $1.20-4$ & \\
\hline 2 & 6 & & $5.56-4$ & $4.91-4$ & $3.86-4$ & $3.51-4$ & & $1.86-4$ & -13 \\
\hline 2 & 7 & $7.35-5$ & $5.72-5$ & $3.92-5$ & $1.13-5$ & $6.22-6$ & $1.52-6$ & $8.14-7$ & 2.5 \\
\hline 2 & 8 & & & & & & & & \\
\hline 2 & 9 & $1.55-4$ & $1.18-4$ & $1.03-4$ & $8.46-5$ & $7.78-5$ & $5.50-5$ & $4.22-5$ & $2.42-13$ \\
\hline 2 & 10 & $7.59-4$ & $7.49-4$ & $7.42-4$ & $7.07-4$ & & & & \\
\hline 2 & 11 & $1.78-4$ & $1.74-4$ & $1.74-4$ & $1.78-4$ & $1.82-4$ & $2.05-4$ & $2.20-4$ & $2.82-4$ \\
\hline 2 & 12 & & $7.73-5$ & $6.79-5$ & $5.70-5$ & $5.27-5$ & $3.72-5$ & $2.83-5$ & $1.62-13$ \\
\hline 2 & 13 & $9.15-4$ & $8.93-4$ & $8.83-4$ & $8.31-4$ & $7.69-4$ & $5.02-4$ & $3.63-4$ & $1.60-6$ \\
\hline 2 & 14 & $1.04-3$ & $9.85-4$ & $9.67-4$ & $9.04-4$ & $8.36-4$ & $5.46-4$ & $3.94-4$ & $6.32-12$ \\
\hline 2 & 15 & $2.22-3$ & $2.23-3$ & $2.27-3$ & $2.43-3$ & $2.60-3$ & $3.97-3$ & $5.18-3$ & $5.31-3$ \\
\hline 2 & 16 & $1.68-3$ & $1.69-3$ & $1.72-3$ & $1.87-3$ & $2.04-3$ & $3.27-3$ & $4.33-3$ & $4.56-3$ \\
\hline 2 & 17 & & & $4.20-4$ & & & & & \\
\hline 2 & 18 & $1.23-4$ & $1.23-4$ & $1.24-4$ & $1.28-4$ & $1.32-4$ & $1.71-4$ & $2.09-4$ & $1.92-4$ \\
\hline 2 & 19 & $1.07-4$ & $1.06-4$ & $1.07-4$ & $1.05-4$ & & & & \\
\hline 3 & 4 & $2.74-4$ & $2.20-4$ & $1.66-4$ & $8.81-5$ & $7.60-5$ & $7.91-5$ & $8.95-5$ & $8.80-5$ \\
\hline 3 & 5 & & $7.02-5$ & $6.01-5$ & $3.01-5$ & $2.22-5$ & & & $3.33-14$ \\
\hline 3 & 6 & $4.08-4$ & $3.06-4$ & $2.52-4$ & $1.65-4$ & $1.43-4$ & $9.54-5$ & $7.29-5$ & $4.03-6$ \\
\hline 3 & 7 & $1.06-3$ & $1.05-3$ & $9.54-4$ & $7.80-4$ & $7.15-4$ & $5.01-4$ & $3.82-4$ & $8.88-14$ \\
\hline 3 & 8 & $8.97-5$ & $6.41-5$ & $5.03-5$ & $3.28-5$ & $2.88-5$ & $1.95-5$ & $1.50-5$ & $6.36-14$ \\
\hline 3 & 9 & $1.58-3$ & $1.45-3$ & $1.38-3$ & $1.34-3$ & $1.34-3$ & $1.45-3$ & $1.53-3$ & $1.98-3$ \\
\hline 3 & 10 & $2.38-4$ & $2.24-4$ & $2.17-4$ & $1.99-4$ & $1.84-4$ & $1.21-4$ & $8.78-5$ & $1.53-6$ \\
\hline 3 & 11 & $2.87-4$ & $2.56-4$ & $2.37-4$ & $2.10-4$ & $1.95-4$ & $1.39-4$ & $1.06-4$ & $4.63-13$ \\
\hline 3 & 12 & $2.42-3$ & $2.09-3$ & $2.01-3$ & $2.00-3$ & $2.04-3$ & $2.31-3$ & $2.49-3$ & $3.07-3$ \\
\hline 3 & 13 & $1.29-3$ & $1.11-3$ & $1.06-3$ & $9.65-4$ & $8.97-4$ & $6.42-4$ & $5.22-4$ & $1.38-4$ \\
\hline 3 & 14 & $2.54-3$ & $2.21-3$ & $2.12-3$ & $1.96-3$ & & $1.56-3$ & $1.48-3$ & $8.04-4$ \\
\hline 3 & 15 & $1.12-3$ & $1.10-3$ & $1.09-3$ & $1.06-3$ & $1.01-3$ & $8.96-4$ & $8.82-4$ & $5.78-4$ \\
\hline 3 & 16 & $7.07-4$ & $7.03-4$ & $7.09-4$ & $7.41-4$ & $7.75-4$ & $1.07-3$ & $1.35-3$ & $1.34-3$ \\
\hline 3 & 17 & $3.72-3$ & $3.70-3$ & $3.72-3$ & $3.80-3$ & $3.88-3$ & $4.83-3$ & $5.83-3$ & $5.33-3$ \\
\hline 3 & 18 & $4.37-3$ & $4.40-3$ & $4.47-3$ & $4.78-3$ & $5.12-3$ & $7.79-3$ & $1.01-2$ & $1.04-2$ \\
\hline 3 & 19 & $1.29-3$ & $1.29-3$ & $1.30-3$ & $1.34-3$ & $1.37-3$ & $1.78-3$ & $2.18-3$ & $2.07-3$ \\
\hline
\end{tabular}



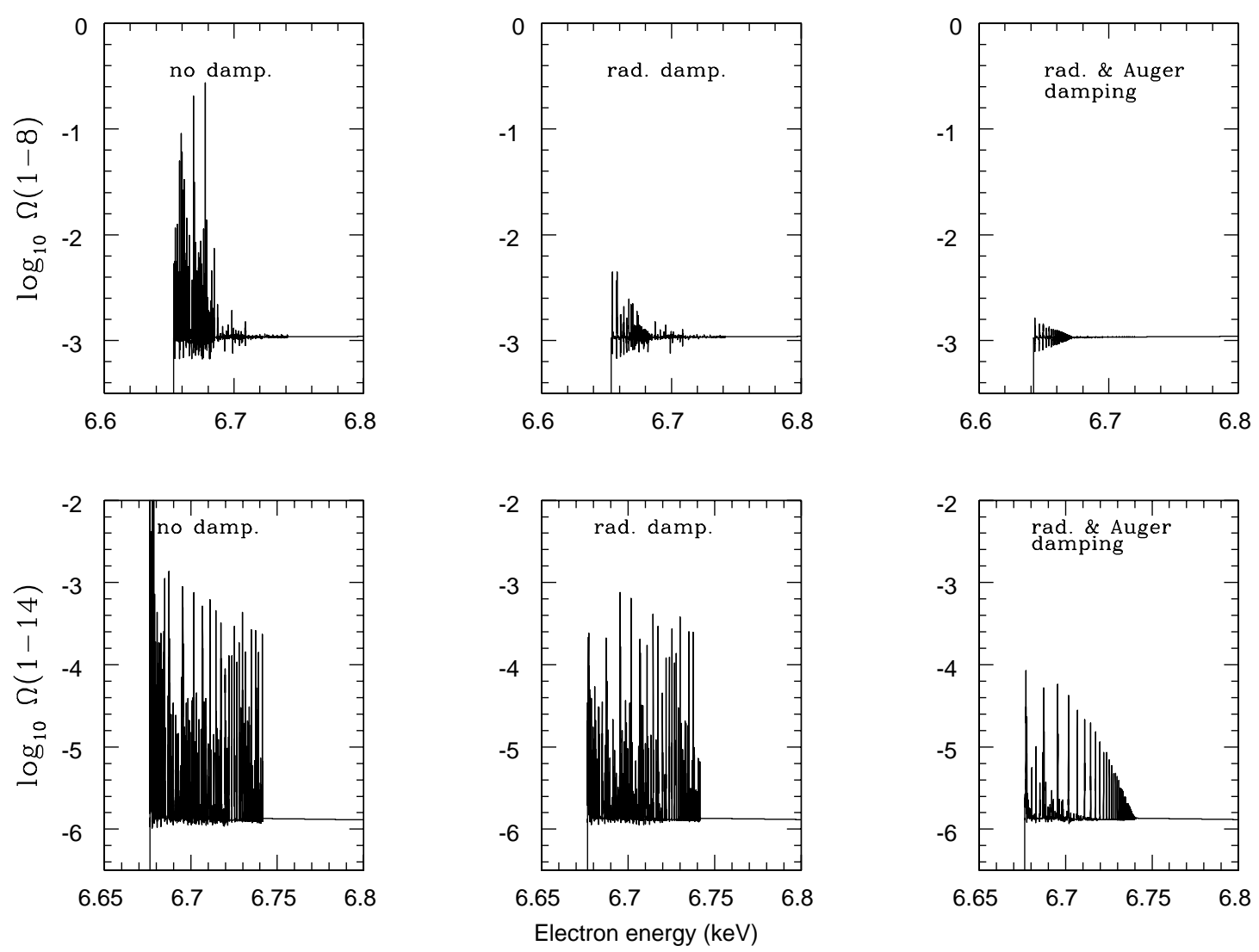

Fig. 5. Comparison of electron impact collision strengths for K-shell excitation in Fe XXIV computed with the BPRM method. The left panels depict collision strengths for the 1-8 and 1-14 transitions computed without damping. The effects of radiation and spectator Auger dampings can be appreciated in the middle and right panels, respectively.

$\Upsilon_{\mathrm{r}}(1)=\frac{4 g f}{\Delta E}$

where $g f$ is the weighted oscillator strength for the transition. Similarly, for a forbidden transition the scaling relations are given by

$T_{\mathrm{r}}=\frac{\kappa T / \Delta E}{\kappa T / \Delta E+c}$

$\Upsilon_{\mathrm{r}}\left(T_{\mathrm{r}}\right)=\Upsilon(T)$

with the following limit points:

$\Upsilon_{\mathrm{r}}(0)=\Omega(0)$

$\Upsilon_{\mathrm{r}}(1)=\Omega_{\mathrm{CB}}$

where $\Omega_{\mathrm{CB}}$ is the Coulomb-Born high-energy limit. The $g f$ and $\Omega_{\mathrm{CB}}$ have been computed with AUTOSTRUCTURE with approximation AST1.

In Fig. 6 the present scaled effective collision strengths for transitions arising from the ground state are compared with previous work. The data by Sampson et al. (1985) and Ballance et al. (2001) have been excluded because the former is practically the same as Goett et al. (1984) while the latter have been superseded by Whiteford et al. (2002). It can be seen that the general agreement is very good: for Whiteford et al. (2002) and our results, it is $\sim 10 \%$ except at low temperatures $\left(<10^{6} \mathrm{~K}\right)$ in the forbidden (1-4) and intersystem (1-6) transitions. The increases are due to the contributions from $n=3$ resonances that have been taken into account by Whiteford et al. which are also significant for transitions with very small $\left(<10^{-5}\right)$ collision strengths. The discrepancies $(\sim 20 \%)$ with the data by Bely-Dubau et al. (1982) in the 1-4 transition are perhaps due to their implementation of the Breit-Pauli corrections.

\section{Inner-shell photoabsorption and photoionization of Fe XXIII}

The inner-shell photoabsorption cross section of the Fe XXIII ground state has been computed with BPRM using the same 19-level Li-like target model described in Sect. 10. As shown in Fig. 7a, the cross section is dominated by a series of symmetric resonances of constant width that cause the smearing of the $\mathrm{K}$ edge. This unusual resonance behavior, as explained by Palmeri et al. (2002), is a consequence of the dominance of $\mathrm{K} \alpha$ and KLL dampings. When such damping is neglected (see Fig. 7b), only the lowest $n=2$ resonance array is accurately represented with our $n=2$ target model whereas the widths of the higher components are markedly underestimated and decrease with $n$ maintaining edge sharpness. Previous close-coupling work on the $\mathrm{K}$ shell photoionization of Be-like Fe (Berrington et al. 1997; Ballance et al. 2001) have neglected Auger damping, and therefore predict narrower high- $n$ resonances and thus a sharp edge. The present total 

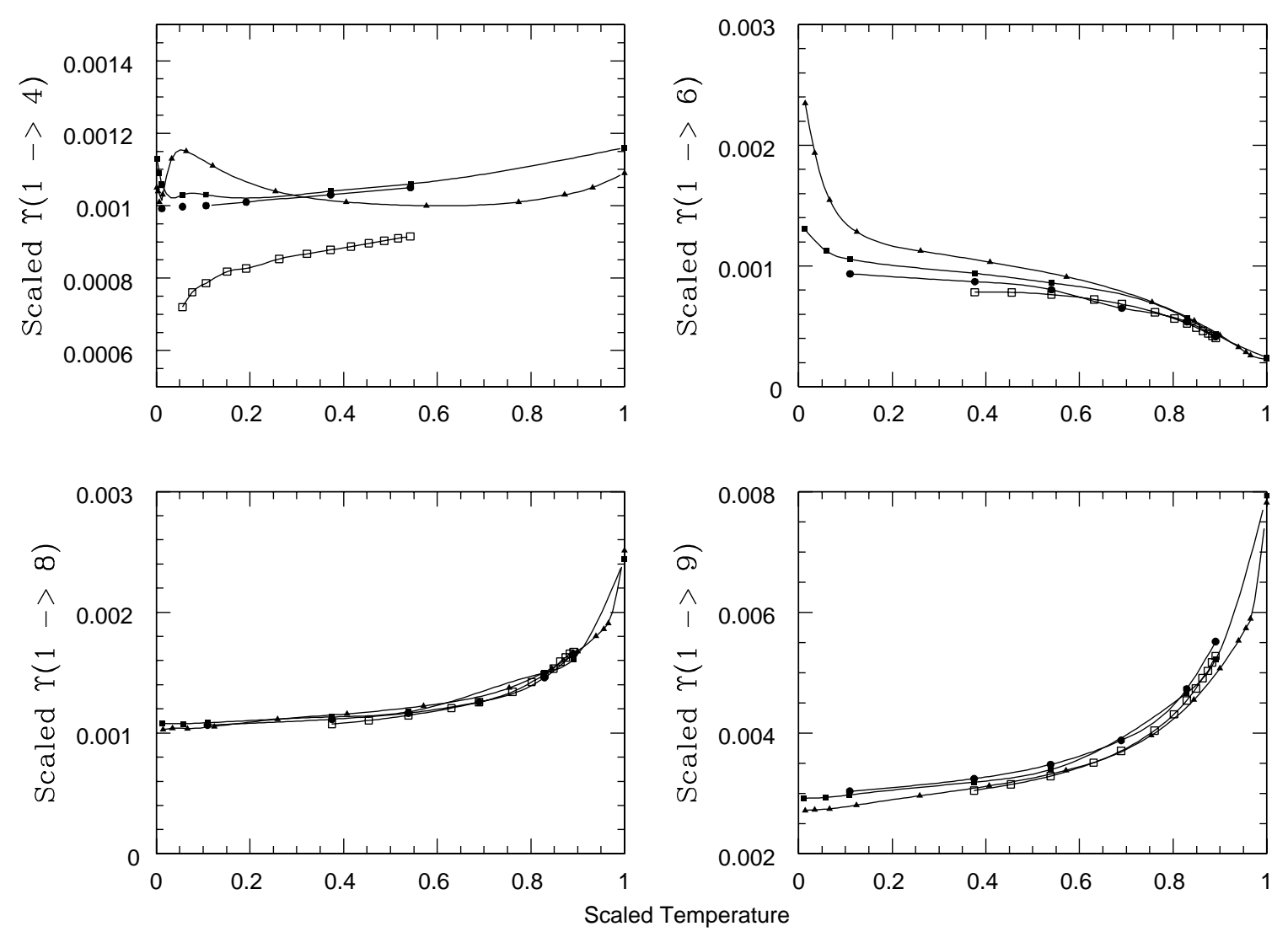

Fig. 6. Comparison of electron impact effective collision strengths using the reduced scales of Burgess \& Tully (1992) (see Sect. 10). Squares: distorted-wave data by Bely-Dubau et al. (1982). Filled circles: Coulomb-Born-Exchange data of Goett et al. (1984). Filled triangles: BPRM calculation by Whiteford et al. (2002). Filled squares: present BPRM results. The discrepancies between the BPRM results at low temperatures are believed to be due to different target representations.

photoabsorption cross section can be accessed online from the TIPTOP $^{1}$ database.

A further key point to make is that when damping is fully taken into account the inner-shell photoabsorption and photoionization processes must be treated separately. In the former, the integrated cross section under the resonance must remain constant in spite of the broadening caused by damping so as to conserve oscillator strength. In the latter, the cross section is actually reduced since radiation damping leads to radiative deexcitation instead of photoionization. Unfortunately, there is as yet no formal procedure to separate the radiative de-excitation component in BPRM.

An alternative method is to compute photoabsorption and photoionization cross sections with AUTOSTRUCTURE by estimating a central-field background cross section and making use of the isolated resonance approximation to compute resonance positions, radiative decay rates and Auger widths for all levels with configurations $1 \mathrm{~s} 2 l 2 l^{\prime} n l^{\prime \prime}$. Assuming Lorentzian profiles, resonances in photoabsorption and photoionization cross sections can be approximated by the expressions

$\sigma^{\mathrm{abs}}=\frac{g f\left(\Gamma_{\mathrm{r}}+\Gamma_{\mathrm{a}}\right)}{\left(E-E_{k}\right)^{2}+1 / 4\left(\Gamma_{\mathrm{r}}+\Gamma_{\mathrm{a}}\right)^{2}}$

\footnotetext{
${ }^{1}$ http://heasarc.gsfc.nasa.gov/topbase
}

and

$\sigma^{\text {ion }}=\frac{g f \Gamma_{\mathrm{a}}}{\left(E-E_{k}\right)^{2}+1 / 4\left(\Gamma_{\mathrm{r}}+\Gamma_{\mathrm{a}}\right)^{2}}$,

where $g f$ is the weighted absorption oscillator strength, $\Gamma_{\mathrm{r}}$ and $\Gamma_{\mathrm{a}}$ are respectively the radiative and Auger widths, and $E$ and $E_{k}$ the photon and resonance energies. In Fig. 8 the photoabsorption and photoionization cross sections calculated with AUTOSTRUCTURE are depicted. The attenuated resonance heights in the photoionization can be appreciated (see Fig. 8b), and a good quantitative resemblance is found for the former with that obtained with BPRM (see Figs. 7a and 8a).

Partial photoionization cross sections of the Fe XXIII ground state leaving the Li-like remnant in a $\mathrm{K}$ vacancy state are displayed in Fig. 9. Only the stronger transitions are included where it is seen that the transition to the $1 \mathrm{~s} 2 \mathrm{~s}^{2}{ }^{2} \mathrm{~S}_{1 / 2}$ level dominates. Since the radiative transition rates for this state are an order of magnitude lower than its Auger width (see Tables 4 and 6), the most probable final state in its decay tree is the ground state of Fe XXV. Therefore, the inner-shell photoionization of the ground state of Fe XXIII yields a double ionization rather than a satellite line. Furthermore, since the $1 \mathrm{~s}^{2} 2 \mathrm{~s} 2 \mathrm{p}{ }^{3} \mathrm{P}_{0}^{0}$ and ${ }^{3} \mathrm{P}_{2}^{0}$ excited states of Fe XXIII are metastable, their photoionization contribution should be in principle included in models. However, unlike the ground state, their 

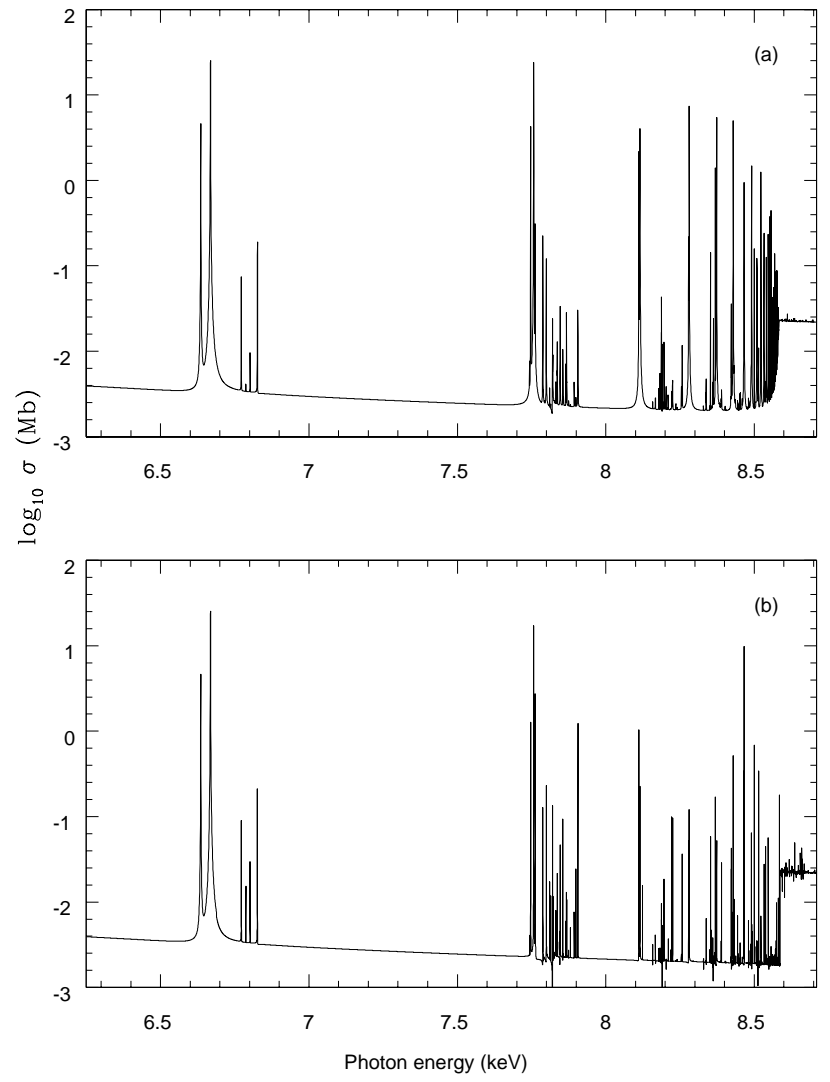

Fig. 7. Total photoabsorption cross section of the ground state of Fe XXIII. The upper panel a) depicts the cross section computed including radiative and spectator-Auger damping effects. The lower panel b) shows the same cross section when these effects are neglected for resonances with $n>2$.

photoionization leaves the ion in $\mathrm{K}$ levels with strong radiative channels that produce satellite lines.

\section{Summary and conclusions}

As a start in a project to compute atomic data for the spectral modeling of $\mathrm{Fe} \mathrm{K}$ lines, we have carried out extensive calculations and comparisons of atomic data for the $\mathrm{K}$ spectrum of Li-like Fe XXIV. The data set includes energy levels, radiative and Auger rates, collision strengths, and total and partial photoionization cross sections. Primary aims have been to select an applicable computational platform and an efficient strategy to generate data sets which are as accurate and complete as possible for other ions of the Fe isonuclear sequence.

We have studied several physical effects, namely orbital representations, core relaxation, CI, relativistic corrections, cancellation, semi-empirical corrections, and the damping of resonances by radiative and spectator Auger decays. For an $N$-electron ion, we have found that the most realistic representation is to have different orbital bases for the $\mathrm{K}$ vacancy states, on the one hand, and for the valence states of the $N$ - and $(N-1)$-electron systems on the other. This is available in HFR, but most other codes use orthogonal orbital bases for computational efficiency. In the case the AUTOSTRUCTURE, which uses a distorted-wave approach to compute Auger rates,

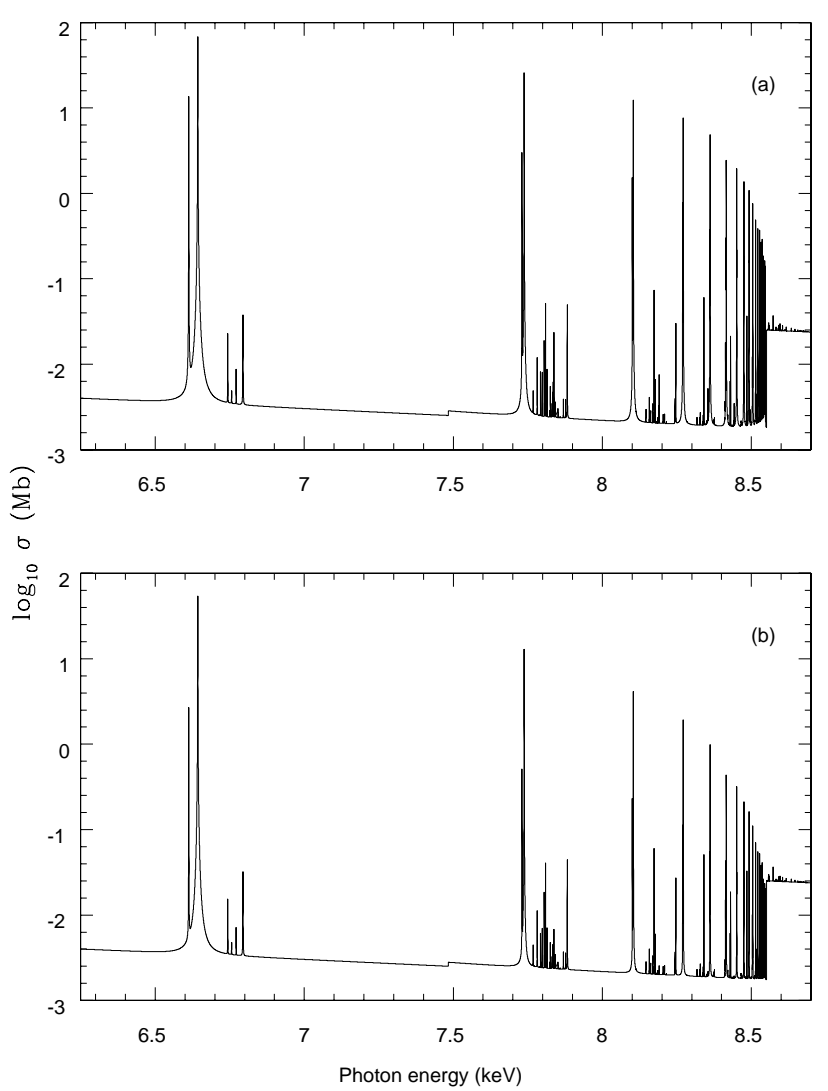

Fig. 8. Comparison between the a) photoabsorption cross section and the b) photoionization cross section of the ground state of Fe XXIII computed with AUTOSTRUCTURE assuming Lorentzian resonance profiles.

orbitals of the $(N-1)$-electron system must then be used. Core relaxation leads to increases in the radiative and Auger widths no larger than $10 \%$.

Level coupling within the $n=2$ complex has been found to be essential, thus seriously questioning the reliability of the atomic model adopted by Lemen et al. (1984). CI from higher complexes contributes negligibly. Contributions from the twobody relativistic operators, both fine structure and non-fine structure, play a conspicuous role in the decay of K-vacancy states of this ion, particularly in the Auger pathways. Electron correlation could be then interpreted as being highly magnetic: bound-free spin-spin effects have been shown to be important within the $n=2$ complex and specially critical for the Auger decay of the metastable $1 \mathrm{~s} 2 \mathrm{~s} 2 \mathrm{p}{ }^{4} \mathrm{P}_{5 / 2}^{0}$ state. This state is also shown to decay radiatively through forbidden M1 and M2 transitions, the former requiring a relativistic corrected transition operator to avoid errors in the line strength of several orders of magnitude. In this highly ionized magnetic scenario, computer programs that do not include a formal numerical implementation of the Breit interaction, or neglect it, have limited applicability. Such is the case of BPRM and HFR. Some of the large discrepancies found for the smaller rates have been attributed to strong cancellation effects, and therefore have been excluded from accuracy ratings. Fine tuning has been found to be a useful option to attain high numerical accuracy, 

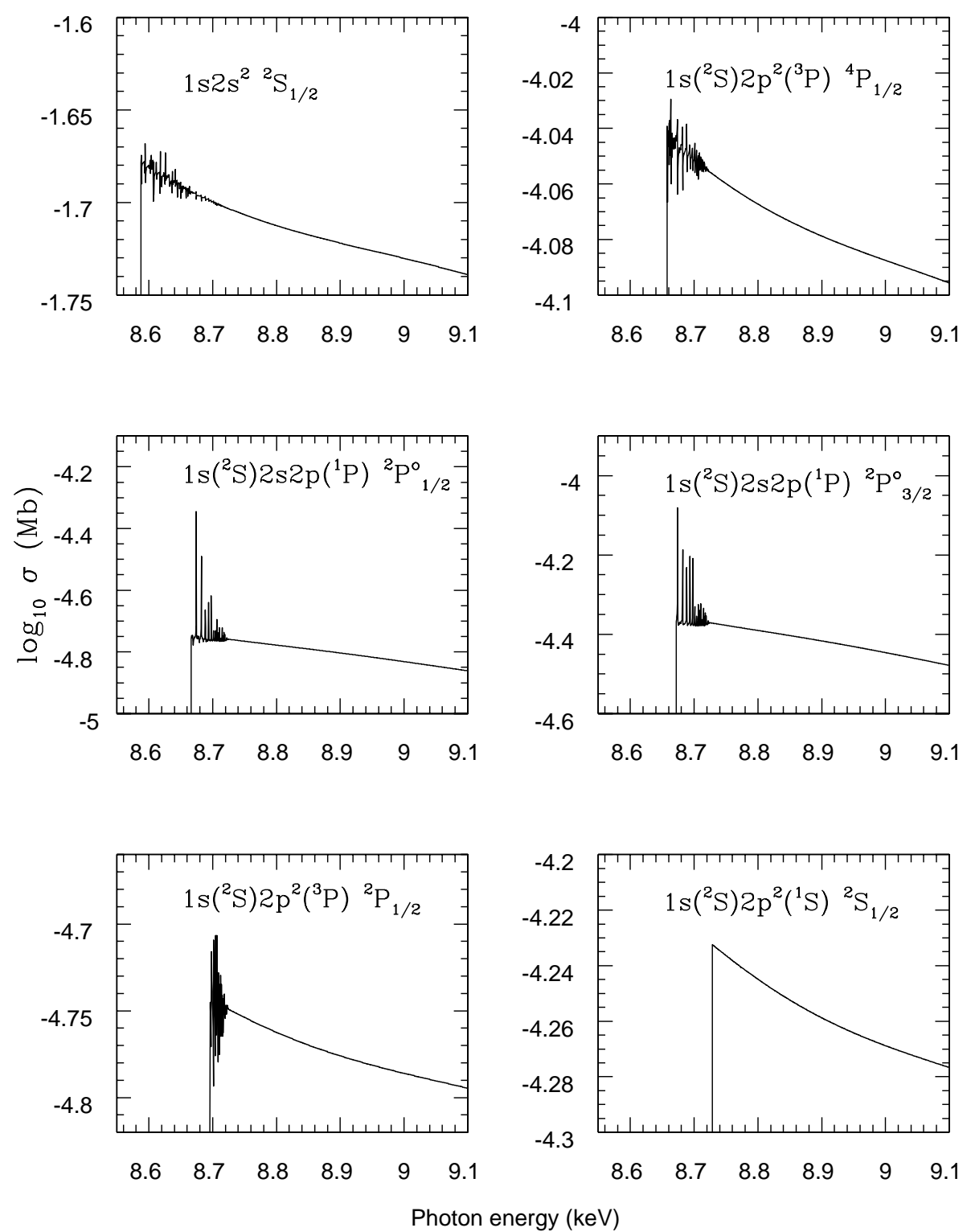

Fig. 9. Partial photoionization cross sections from the ground level of Fe XXIII leaving Fe XXIV in a K-vacancy state.

particularly for line identification and to render intersystem couplings that can be very sensitive to level separations.

The present AUTOSTRUCTURE calculations are an independent validation and refinement of that performed in COR; the level of agreement found at the different stages confirms this assertion. The excellent accord also obtained with the radiative rates by SAF allows us to suggest a ranking of $10 \%$ for the present $A$-values. On the other hand, the fairly large discrepancies with the SAF Auger rates are believed to be caused by their approximate treatment of the Breit interaction in terms of screening constants. We therefore rank the present autoionization data at better than $15 \%$. We can also conclude by comparing with SAF that the attained precision for the K-vacancy level energies of $\pm 2 \mathrm{eV}$ is a representative lower bound for current numerical methods. This however implies fine tuning that relies on spectroscopic measurements. Since complete experimental level structures are not available for most systems, further experiments would be welcome.
Both radiative and spectator Auger dampings have been taken into account in the calculation of K-shell photoabsorption and electron excitation processes. In photoabsorption, resonances converging to the $\mathrm{K}$ threshold acquire a peculiar behavior that leads to edge smearing which, as discussed by Palmeri et al. (2002), has diagnostic potential in astrophysical plasmas. With regards to electron excitation, resonances are practically washed out, thus simplifying target modeling or the choice of a suitable numerical approach. This assertion is supported by the good agreement $(10 \%)$ of the present excitation rates with the Coulomb-Born-Exchange results of Goett et al. (1984) and with those in the $R$-matrix calculation by Whiteford et al. (2002) who used a more refined target. We have also found that the ground state of Fe XXIII is mainly photoionized to the $1 \mathrm{~s} 2 \mathrm{~s}^{2}{ }^{2} \mathrm{~S}_{1 / 2} \mathrm{~K}$ level of Fe XXIV which rapidly autoionizes rather than fluoresces. Thus $\mathrm{K} \alpha$ emission from a $\mathrm{Fe} \mathrm{Li-like} \mathrm{ion}$ is mainly the result of electron impact excitation and dielectronic recombination. 
The approach we have taken in our study of the Fe XXIV $\mathrm{K}$ vacancy states is based on the use and comparison of results of several computational platforms. We conclude that, among the codes available to us, for Auger, radiative and structure calculations in this ion the most accurate results are obtained using AUTOSTRUCTURE plus TEC. For electron impact excitation we find good agreement between BPRM and the CBE results, owing to the fact that damping reduces the importance of resonances. For scattering of both electrons and photons we conclude therefore that BPRM (including damping) is the platform of choice. The multi-platform approach has proven to be useful in elucidating the physics involved, and has been used previously by COR and SAF and more recently by Savin et al. (2002). We have also produced what we feel is, on the whole, an accurate and consistent dataset for atomic data for the Fe XXIV K vacancy states, although a few of our computed quantities are in less good agreement with experiment than are those of SAF or MCDF. This work has given us confidence in the use of these tools, and the multi-platform approach, when applied to the rest of the $\mathrm{Fe}$ isonuclear sequence.

The present data sets can be accessed on line from the TIPTOP $^{2}$ database.

Acknowledgements. We are indebted to Dr. Nigel Badnell from the University of Strathclyde, UK, for invaluable discussions regarding the AUTOSTRUCTURE options, Auger processes in general and the peculiar decay properties of the K-vacancy metastable state of this ion. Also to Dr. Marguerite Cornille, Observatoire de Meudon, France, for details about the COR and SAF calculations. CM acknowledges a Senior Research Associateship from the National Research Council, and MAB support from FONACIT, Venezuela, under contract No. S120011000912. Support for this research was provided in part by a grant from the NASA Astrophysics Theory Program.

\section{References}

Badnell, N. R. 1986, J. Phys. B, 19, 3827

Badnell, N. R. 1997, J. Phys. B, 30, 1

Ballance, C. P., Badnell, N. R., \& Berrington, K. A. 2001, J. Phys. B, 34,3287

Barr, P., White, N. E., \& Page, C. G. 1985, MNRAS, 216, 65P

Beiersdorfer, P., Bitter, M., von Goeler, S., et al. 1989, Phys. Rev. A, 40, 150

Beiersdorfer, P., Phillips, T., Jacobs, V. L., et al. 1993, ApJ, 409, 846

Beiersdorfer, P., Phillips, T. W., Wong, K. L., et al. 1992, Phys. Rev. A, 46,3812

Bely-Dubau, F., Dubau, J., Faucher, P., et al. 1982, MNRAS, 198, 239

Berrington, K. A., \& Ballance, C. 2001, J. Phys. B, 34, 2697

Berrington, K. A., Burke, P. G., Butler, K., et al. 1987, J. Phys. B, 20, 6379

Berrington, K. A., Burke, P. G., Chang, J. J., et al. 1974, Comput. Phys. Commun., 8, 149

Berrington, K. A., Burke, P. G., Le Dourneuf, et al. 1978, Comput. Phys. Commun., 14, 367

Berrington, K. A., Burke, P. G., Eissner, W., et al. 1995, Comput. Phys. Commun., 92, 290

Berrington, K., Quigley, L., \& Zhang, H. L. 1997, J. Phys. B, 30, 5409

Breuckmann, B. 1979, J. Phys. B, 12, L609
Burgess, A., \& Tully, J. A. 1992, A\&A, 254, 436

Burke, P. G., Hibbert, A., \& Robb, W. D. 1971, J. Phys. B, 4, 153

Burke, P. G., \& Seaton, M. J. 1971, Meth. Comp. Phys., 10, 1

Chen, M. H. 1986, At. Data Nucl. Data Tables, 34, 301

Chen, M. H., Crasemann, B., \& Mark, H. 1981, Phys. Rev. A, 24, 1852

Cowan, R. D. 1981, The Theory of Atomic Structure and Spectra (Berkeley, CA: University of California Press)

Decaux, V., \& Beiersdorfer, P. 1993, Phys. Scr., T47, 80

Decaux, V., Beiersdorfer, P., Kahn, S. M., et al. 1997, ApJ, 482, 1076

Decaux, V., Beiersdorfer, P., Osterheld, A., et al. 1995, ApJ, 443, 464

Donnelly, D. W., Bell, K. L., Scott, M. P., et al. 2000, ApJ, 531, 1168

Drake, G. W. F. 1971, Phys. Rev. A, 3, 908

Dubau, J., \& Loulergue, M. 1981, Phys. Scr., 23, 136

Eissner, W., Jones, M., \& Nussbaumer, H. 1974, Comput. Phys. Commun., 8, 270

Eissner, W., \& Nussbaumer, H. 1969, J. Phys. B, 2, 1028

Eissner, W., \& Zeippen, C. J. 1981, J. Phys. B, 14, 2125

Gabriel, A. H. 1972, MNRAS, 160, 99

Goett, S. J., Sampson, D. H., \& Clark, R. E. H. 1984, ApJS, 54, 115

Gorczyca, T. W., \& Badnell, N. R. 1996, J. Phys. B, 29, L283

Gorczyca, T. W., \& Badnell, N. R. 2000, J. Phys. B, 33, 2511

Howat, G. 1978, J. Phys. B, 11, 1589

Howat, G., Åberg, T., \& Goscinski, O. 1978, J. Phys. B, 11, 1575

Jacobs, V. L., \& Rozsnyai, B. F. 1986, Phys. Rev. A, 34, 216

Jacobs, V. L., Davis, J., Rozsnyai, B. F., et al. 1980, Phys. Rev. A, 21, 1917

Jacobs, V. L., Doschek, G. A., Seely, J. F., et al. 1989, Phys. Rev. A, 39,2411

Kato, T., Safronova, U. I., Shlyaptseva, A. S., et al. 1997, At. Data Nucl. Data Tables, 67, 225

Lemen, J. R., Phillips, K. J. H., Cowan, R. D., et al. 1984, A\&A, 135, 313

Marshall, F. E., Mushotzky, R. F., Petre, R., et al. 1993, ApJ, 419, 301

Nahar, S. N., Pradhan, A. K., \& Zhang, H. L. 2001, Phys. Rev. A, 63, 060701

Palmeri, P., Mendoza, C., Kallman, T. R., et al. 2002, ApJ, 577, L119

Pradhan, A. K., \& Gallagher, J. W. 1992, At. Data Nucl. Data Tables, 52,227

Pravdo, S. H., Becker, R. H., Boldt, E. A., et al. 1977, ApJ, 215, L61

Quigley, L., \& Berrington, K. 1996, J. Phys. B, 29, 4529

Quigley, L., Berrington, K., \& Pelan, J. 1998, Comput. Phys. Commun., 114, 225

Robicheaux, F., Gorczyca, T. W., Pindzola, M. S., et al. 1995, Phys. Rev. A, 52, 1319

Safronova, U. I., \& Shlyaptseva, A. S. 1996, Phys. Scr., 54, 254

Sampson, D. H., Goett, S. J., Petrou, G. V., et al. 1985, At. Data Nucl. Data Tables, 32, 343

Sanford, P., Mason, K. O., \& Ives, J. 1975, MNRAS, 173, 9P

Savin, D. W., Behar, E., Kahn, S. M., et al. 2002, ApJS, 138, 337

Scott, N. S., \& Burke, P. G. 1980, J. Phys. B, 13, 4299

Scott, N. S., \& Taylor, K. T. 1982, Comput. Phys. Commun., 25, 349

Seely, J. F., Feldman, U., \& Safronova, U. I. 1986, ApJ, 304, 838

Serlemitsos, P. J., Boldt, E. A., Holt, S. S., et al. 1973, ApJ, 184, L1

Serlemitsos, P. J., Smith, B. W., Boldt, E. A., et al. 1977, ApJ, 211, L63

Shirai, T., Sugar, J., Musgrove, A., et al. 2000, J. Phys. Chem. Ref. Data, Monograph, 8

Tanaka, Y., Nandra, K., Fabian, A. C., et al. 1995, Nature, 375, 659

Whiteford, A. D., Badnell, N. R., Ballance, C. P., et al. 2002, J. Phys. B, 35, 3729

\footnotetext{
${ }^{2}$ http://heasarc.gsfc.nasa.gov/topbase
} 